\title{
LYAPUNOV INVERSE ITERATION FOR IDENTIFYING HOPF BIFURCATIONS IN MODELS OF INCOMPRESSIBLE FLOW*
}

\author{
HOWARD C. ELMAN ${ }^{\dagger}$, KARL MEERBERGEN $^{\ddagger}$, ALASTAIR SPENCE $^{\S}$, AND \\ MINGHAO WU
}

\begin{abstract}
The identification of instability in large-scale dynamical systems caused by Hopf bifurcation is difficult because of the problem of identifying the rightmost pair of complex eigenvalues of large sparse generalized eigenvalue problems. A new method developed in [K. Meerbergen and A. Spence, SIAM J. Matrix Anal. Appl., 31 (2010), pp. 1982-1999] avoids this computation, instead performing an inverse iteration for a certain set of real eigenvalues that requires the solution of a large-scale Lyapunov equation at each iteration. In this study, we refine the Lyapunov inverse iteration method to make it more robust and efficient, and we examine its performance on challenging test problems arising from fluid dynamics. Various implementation issues are discussed, including the use of inexact inner iterations and the impact of the choice of iterative solution for the Lyapunov equations, and the effect of eigenvalue distribution on performance. Numerical experiments demonstrate the robustness of the algorithm.
\end{abstract}

Key words. linear stability analysis, Hopf bifurcation, inverse iteration, Lyapunov solvers

AMS subject classifications. 65F15, 65F $18,37 \mathrm{M} 20$

DOI. $10.1137 / 110827600$

1. Introduction. Consider the dynamical system

$$
\mathbf{M} u_{t}=f(u, \alpha)
$$

where $f: \mathbb{R}^{n} \times \mathbb{R} \mapsto \mathbb{R}^{n}$ is a nonlinear mapping, $u \in \mathbb{R}^{n}$ is the state variable (velocity, pressure, temperature, etc.), $\mathbf{M} \in \mathbb{R}^{n \times n}$, and $\alpha$ is a parameter. Such problems arise from finite element discretization of partial differential equations (PDEs) where the matrix $\mathbf{M}$ is usually called the mass matrix and could be singular. The dimension of the discretization, $n$, is usually large, especially for three-dimensional PDEs. Let $u$ denote the steady-state solution to (1.1), i.e., $u_{t}=0$. We are interested in the stability of $u$ : if a small perturbation $\delta(0)$ is introduced to $u$ at time $t=0$, does $\delta(t)$ grow with time, or does it decay? Let the solution path of the equilibrium equation $f(u, \alpha)=0$ be the following set: $\mathcal{S}=\{(u, \alpha) \mid f(u, \alpha)=0\}$. $\mathcal{S}$ can be computed using numerical continuation techniques (see, for example, [12]). It is often the case that as

*Submitted to the journal's Methods and Algorithms for Scientific Computing section March 16, 2011; accepted for publication (in revised form) March 13, 2012; published electronically June 12, 2012. This work was supported in part by the U.S. Department of Energy under grant DEFG0204ER25619; the U.S. National Science Foundation under grant CCF0726017; the Belgian Network DYSCO (Dynamical Systems, Control, and Optimization), funded by the Interuniversity Attraction Poles Programme, initiated by the Belgian State Science Policy Office; and the Research Council K.U. Leuven grants CoE EF/05/006 and OT/10/038.

http://www.siam.org/journals/sisc/34-3/82760.html

$\dagger$ Department of Computer Science and Institute for Advanced Computer Studies, University of Maryland, College Park, MD 20742 (elman@cs.umd.edu).

${ }^{\ddagger}$ Department of Computer Science, Katholieke Universiteit Leuven, 3001 Heverlee (Leuven), Belgium (Karl.Meerbergen@cs.kuleuven.be).


(a.spence@maths.bath.ac.uk).

IApplied Mathematics \& Statistics, and Scientific Computation Program, Department of Mathematics, University of Maryland, College Park, MD 20742 (mwu@math.umd.edu).

A1584 
the parameter $\alpha$ varies, there exists a critical point $\left(u_{c}, \alpha_{c}\right) \in \mathcal{S}$ at which the steadystate solution $u$ changes from being stable to unstable. An important problem in applications is to find this critical parameter value $\alpha_{c}$ assuming that (a portion of) $\mathcal{S}$ is known. For a fixed value of $\alpha$, linear stability of the steady-state solution is determined by the spectrum of the eigenvalue problem

$$
\mathcal{J} x=\mu \mathbf{M} x,
$$

where $\mathcal{J}=\frac{\partial f}{\partial u}(u(\alpha), \alpha)$ is the Jacobian matrix of $f$ evaluated at $\alpha$. If all the eigenvalues of (1.2) have strictly negative real part, then $u$ is a stable steady solution; if some eigenvalues of (1.2) have nonnegative real part, then $u$ is unstable. Therefore, a change of stability can be detected by monitoring the rightmost eigenvalues in the complex plane of (1.2) while marching along $\mathcal{S}$.

A steady-state solution may lose its stability in one of two ways: either the rightmost eigenvalue of (1.2) is real and passes through zero from negative to positive as $\alpha$ varies, as in the case of a fold point or a symmetry-breaking bifurcation point [12], or (1.2) has a complex pair of rightmost eigenvalues and they cross the imaginary axis as $\alpha$ varies, which leads to a Hopf bifurcation with the consequent birth of periodic solutions of (1.1). The first case is easier to detect because the rightmost eigenvalue is real and close to zero and there are many methods that are reliable for computing eigenvalues near a target, for example, the shift-and-invert Arnoldi method. However, methods like shift-and-invert Arnoldi may fail to detect the instability in the second case unless good estimates of the rightmost eigenvalues are available, which is not the case in general.

Guckenheimer and Myers [13] and Guckenheimer, Myers, and Sturmfels [14] proposed a method that computes Hopf points without computing the rightmost eigenvalues of (1.2) with $\mathbf{M}=\mathbf{I}$, the identity matrix of order $n$. Their method is based on the following property of the Kronecker sum $\mathcal{J} \otimes \mathbf{I}+\mathbf{I} \otimes \mathcal{J}$ (assuming $\mathcal{J}$ is nonsingular): it has a double zero eigenvalue if and only if $\mathcal{J} x=\mu x$ has one and only one pair of eigenvalues that sums to zero. The method uses the equilibrium equation $f(u, \alpha)=0$ together with the condition $\operatorname{det}(\mathcal{J}(u, \alpha) \otimes \mathbf{I}+\mathbf{I} \otimes \mathcal{J}(u, \alpha))=0$, where $\mathcal{J}(u, \alpha)$ denotes the Jacobian matrix $\frac{\partial f}{\partial u}(u, \alpha)$, as the defining system of Hopf points, and Newton's method is used to solve for the roots $\left(u_{c}, \alpha_{c}\right)$ of this system. Unfortunately, this algorithm requires the solution of linear systems of order $n^{2}$, where $n$ is the order of $\mathcal{J}$ and $\mathbf{M}$; therefore it is not suitable for large-scale problems in which $n$ is already large. Nonetheless, based on this approach, Meerbergen and Spence [18] proposed a method that estimates the critical parameter value without computing the rightmost eigenvalues of (1.2) or working with the Kronecker sum of order $n^{2}$ directly. Estimates of the rightmost eigenvalues can be obtained as by-products.

The aims of this paper are (i) to further understand and refine the method discussed in [18] to make it more efficient and reliable, (ii) to test it on more challenging examples arising in fluid dynamics, and (iii) to provide a discussion of the efficiency of large-scale Lyapunov solvers arising from this approach.

Throughout this paper, we will focus on the case in which the stability of the steady-state solution is lost to a Hopf bifurcation point, although the ideas we study are also applicable to the case where instability is caused by a real eigenvalue crossing the imaginary axis. Assume that $\left(u_{0}, \alpha_{0}\right)$ is a stable point on the solution path $\mathcal{S}$ and that the Hopf point $\left(u_{c}, \alpha_{c}\right)$ at which stability is lost lies in its neighborhood. The Jacobian matrix at any point $(u, \alpha)$ in the neighborhood of $\left(u_{0}, \alpha_{0}\right)$ can be approximated as $\mathcal{J}(\alpha) \approx \mathcal{J}\left(\alpha_{0}\right)+\left(\alpha-\alpha_{0}\right) \frac{d \mathcal{J}}{d \alpha}\left(\alpha_{0}\right)=\mathbf{A}+\lambda_{\alpha} \mathbf{B}$, where $\mathbf{A}, \mathbf{B}$ are known and 
$\lambda_{\alpha}$ is an unknown quantity that characterizes the distance from $\left(u_{0}, \alpha_{0}\right)$ to $(u, \alpha)$. In particular, the Jacobian matrix at the Hopf point can be approximated by $\mathbf{A}+\lambda_{c} \mathbf{B}$, where $\lambda_{c}=\alpha_{c}-\alpha_{0}$. The critical value $\alpha_{c}$ can then be approximated by computing $\lambda_{c}$. We assume for simplicity that $\mathcal{J}(\alpha)=\mathbf{A}+\lambda_{\alpha} \mathbf{B}$ in the neighborhood of $\left(u_{0}, \alpha_{0}\right)$. Consider the parameterized eigenvalue problem

$$
(\mathbf{A}+\lambda \mathbf{B}) x=\mu \mathbf{M} x .
$$

When $\lambda=\lambda_{c}$, (1.3) has a conjugate pair of pure imaginary rightmost eigenvalues $(\beta i,-\beta i)$ with $\beta>0$. Therefore, $\lambda_{c}$ is the value of $\lambda$ closest to zero such that (1.3) has a pair of eigenvalues that sums to zero. Using the equivalence between equations involving Kronecker products and linear matrix equations, it is shown in [18] that $\lambda_{c}$ is also the parameter closest to zero such that

$$
\mathbf{M} Z \mathbf{A}^{T}+\mathbf{A} Z \mathbf{M}^{T}+\lambda\left(\mathbf{M} Z \mathbf{B}^{T}+\mathbf{B} Z \mathbf{M}^{T}\right)=0,
$$

where $Z \in \mathbb{R}^{n \times n}$ is nonzero. Once $\lambda_{c}$ is computed from (1.4), the critical parameter value $\alpha_{c}$ can be estimated as $\alpha_{0}+\lambda_{c}$, and a corresponding estimate of $\beta i$ can also be found easily. These estimates could be used as starting values in an algorithm for the accurate calculation of a Hopf point (see [12]).

Consider a special case of (1.1), the Navier-Stokes equations governing viscous incompressible flow,

$$
\begin{aligned}
u_{t} & =\nu \nabla^{2} u-u \cdot \nabla u-\nabla p, \\
0 & =\nabla \cdot u,
\end{aligned}
$$

subject to appropriate boundary conditions, where $\nu$ is the kinematic viscosity, $u$ is the velocity, and $p$ is the pressure. The viscosity $\nu$ is a natural candidate for $\alpha$. In the literature, properties of a flow are usually characterized by the Reynolds number (denoted by $R e$ ), a dimensionless quantity proportional to $\frac{1}{\nu}$. For convenience in our exposition, we will sometimes refer to the Reynolds number instead of the viscosity. Mixed finite element discretization of (1.5) gives rise to the following Jacobian matrix and mass matrix [9]:

$$
\mathbf{A}=\left[\begin{array}{cc}
F & B^{T} \\
B & 0
\end{array}\right], \quad \mathbf{M}=\left[\begin{array}{cc}
-G & 0 \\
0 & 0
\end{array}\right] \in \mathbb{R}^{n \times n}
$$

where $n=n_{u}+n_{p}, n_{u}>n_{p}, F \in \mathbb{R}^{n_{u} \times n_{u}}, B \in \mathbb{R}^{n_{p} \times n_{u}}$, and $G \in \mathbb{R}^{n_{u} \times n_{u}}$ is symmetric positive definite. Matrices $F, B, G$ are sparse, and $n$ is usually large. In this paper, we apply the method proposed in [18] to detect the Hopf point at which a steady-state solution of (1.5) loses its stability.

The plan for the rest of the paper is as follows. In section 2, we review the Lyapunov inverse iteration method proposed by Meerbergen and Spence in [18]. In section 3, we discuss a block Krylov method for solving large-scale Lyapunov equations with a low-rank right-hand side, and we propose an efficient way to truncate the computed solution. The main contributions of this paper are in the following two sections. In section 4, we propose an inverse iteration with inexact Lyapunov solvers, which is based on the ideas in [19]. In section 5, the method proposed in section 4 is applied to detect Hopf bifurcation in two incompressible flows and numerical results are presented; in addition, alternative Lyapunov solvers are discussed and compared with the Krylov method of section 3. Finally, in section 6, we make some concluding observations. 
2. Review of the Lyapunov inverse iteration. In this section we review the algorithm for detecting Hopf points proposed in [18] and the mathematical theory on which the algorithm is built. The following theorem is the main theoretical motivation for the techniques in [18].

THEOREM 2.1. Assume $\mathbf{M}$ is nonsingular, and assume $\mu_{1}, \mu_{2}\left(\mu_{1} \neq \mu_{2}\right)$ are simple eigenvalues of (1.2) whose corresponding eigenvectors are $x_{1}, x_{2}$. The following two statements are equivalent:

1. zero is a double eigenvalue of $\mathcal{J} \otimes \mathbf{M}+\mathbf{M} \otimes \mathcal{J}$ that corresponds to the eigenvector $\xi_{1} x_{1} \otimes x_{2}+\xi_{2} x_{2} \otimes x_{1}$ for any $\xi_{1}, \xi_{2} \in \mathbb{C}$;

2. $\left(\mu_{1}, \mu_{2}\right)$ is the only pair of eigenvalues of (1.2) that sums to zero.

Proof. Since $\mathbf{M}$ is nonsingular, by properties of Kronecker products, $\mathbf{M} \otimes \mathbf{M}$ is nonsingular. Also using properties of Kronecker products, we can show that

$$
(\mathbf{M} \otimes \mathbf{M})^{-1}(\mathcal{J} \otimes \mathbf{M}+\mathbf{M} \otimes \mathcal{J})\left(x_{i} \otimes x_{j}\right)=\left(\mu_{i}+\mu_{j}\right)\left(x_{i} \otimes x_{j}\right) .
$$

Thus, the eigenpairs of $(\mathbf{M} \otimes \mathbf{M})^{-1}(\mathcal{J} \otimes \mathbf{M}+\mathbf{M} \otimes \mathcal{J})$ are $\left(\mu_{i}+\mu_{j}, \xi_{i} x_{i} \otimes x_{j}+\xi_{j} x_{j} \otimes x_{i}\right)$ $(i, j=1,2, \ldots, n)$ for any $\xi_{i}, \xi_{j} \in \mathbb{C}$.

We first prove statement 2 given statement 1 . Note that $\mathcal{J} \otimes \mathbf{M}+\mathbf{M} \otimes \mathcal{J}$ and $(\mathbf{M} \otimes \mathbf{M})^{-1}(\mathcal{J} \otimes \mathbf{M}+\mathbf{M} \otimes \mathcal{J})$ have the same null space. Thus, if statement 1 is true, zero is also a double eigenvalue of $(\mathbf{M} \otimes \mathbf{M})^{-1}(\mathcal{J} \otimes \mathbf{M}+\mathbf{M} \otimes \mathcal{J})$ with the eigenvector $\xi_{1} x_{1} \otimes x_{2}+\xi_{2} x_{2} \otimes x_{1}$. By $(2.1),\left(\mu_{1}, \mu_{2}\right)$ is the only pair of eigenvalues of (1.2) that sums to zero.

Now assume statement 2 is true. Since $\left(\mu_{1}, \mu_{2}\right)\left(\mu_{1} \neq \mu_{2}\right)$ is the only pair of eigenvalues of (1.2) that sums to zero and both $\mu_{1}, \mu_{2}$ are simple, by (2.1), zero is a double eigenvalue of $(\mathbf{M} \otimes \mathbf{M})^{-1}(\mathcal{J} \otimes \mathbf{M}+\mathbf{M} \otimes \mathcal{J})$ with the eigenvector $\xi_{1} x_{1} \otimes x_{2}+$ $\xi_{2} x_{2} \otimes x_{1}$. Since $\mathcal{J} \otimes \mathbf{M}+\mathbf{M} \otimes \mathcal{J}$ and $(\mathbf{M} \otimes \mathbf{M})^{-1}(\mathcal{J} \otimes \mathbf{M}+\mathbf{M} \otimes \mathcal{J})$ have the same null space, statement 1 follows immediately.

We continue to assume that $\mathbf{M}$ is nonsingular. In addition, assume that when $\lambda=\lambda_{c}$, the rightmost eigenvalues of (1.3) $(\beta i$ and $-\beta i)$ are simple and there are no other eigenvalues lying on the imaginary axis. Let $v$ and $\bar{v}$ be the eigenvectors corresponding to $\beta i$ and $-\beta i$. Since $\lambda_{c}$ is the parameter closest to zero such that (1.3) has a pair of eigenvalues that sums to zero, according to Theorem $2.1, \lambda_{c}$ is the parameter closest to zero such that $(\mathbf{A}+\lambda \mathbf{B}) \otimes \mathbf{M}+\mathbf{M} \otimes(\mathbf{A}+\lambda \mathbf{B})$ has a zero eigenvalue. Alternatively, $\lambda_{c}$ is the eigenvalue closest to zero for the $n^{2} \times n^{2}$ generalized eigenvalue problem

$$
\left(\Delta_{1}+\lambda \Delta_{0}\right) z=0
$$

where

$$
\begin{aligned}
& \Delta_{1}=\mathbf{A} \otimes \mathbf{M}+\mathbf{M} \otimes \mathbf{A}, \\
& \Delta_{0}=\mathbf{B} \otimes \mathbf{M}+\mathbf{M} \otimes \mathbf{B} .
\end{aligned}
$$

Note that by Theorem 2.1, the eigenvector corresponding to $\lambda_{c}$ is $z_{c}=\xi_{1} v \otimes \bar{v}+\xi_{2} \bar{v} \otimes v$. Therefore, finding $\lambda_{c}$, the quantity that allows us to estimate the critical parameter value $\alpha_{c}$, is equivalent to finding the eigenvalue of (2.2) with smallest modulus. One standard approach for computing this eigenvalue is to use an iterative method such as inverse iteration for (2.2). This approach is obviously impractical for large-scale problems since inverse iteration requires solution of linear systems with coefficient matrix $\Delta_{1}$, which has order $n^{2}$. We can use properties of Kronecker products to rewrite (2.2) into a linear equation of $n \times n$ matrices. In particular, let $Z \in \mathbb{R}^{n \times n}$ 
be such that $z=\operatorname{vec}(Z)$ (see [16, p. 244]). Then it is known (see [16, p. 255]) that (2.2) is equivalent to (1.4). Therefore, finding $\lambda$ with smallest modulus for (2.2) is equivalent to finding $\lambda$ with smallest modulus for (1.4). Because of the relationship between (2.2) and (1.4), we will refer to $\lambda$ as an eigenvalue and $Z$ as an eigenvector of (1.4). The following theorem from [18] describes the properties of $Z$.

TheOREm 2.2. Assume that $\lambda$ is a real eigenvalue of (2.2). If (1.3) has eigenpairs $(\beta i, v)$ and $(-\beta i, \bar{v})(\beta>0)$ and no other eigenvalues on the imaginary axis, then (1.4) has a real symmetric eigenvector of rank two, namely, $Z=v v^{*}+\bar{v} v^{T}$, which is unique up to a scalar factor and is semidefinite, and a unique skew-symmetric eigenvector of rank two, namely, $Z=v v^{*}-\bar{v} v^{T}$.

It is suggested in [18] that we should restrict our computation to the real symmetric eigenspace of (1.4). Under this restriction, the eigenvalue of interest, $\lambda_{c}$, is simple. The corresponding eigenvector, which is symmetric and of rank two, has a natural representation in the form of a truncated eigenvalue decomposition $Z_{c}=\mathcal{V} \mathcal{D} \mathcal{V}^{T}$, where $\mathcal{V} \in \mathbb{R}^{n \times 2}$ is orthonormal and $\mathcal{D} \in \mathbb{R}^{2 \times 2}$ is diagonal. By Theorem 2.2, $\operatorname{span}\{\mathcal{V}\}=\operatorname{span}\{v, \bar{v}\}$. Therefore, once we find $\lambda_{c}$ and its eigenvector $Z_{c}$ for (1.4), the rightmost eigenvalues of (1.3) can be found easily by solving the $2 \times 2$ problem

$$
\mathcal{V}^{T}\left(\mathbf{A}+\lambda_{c} \mathbf{B}\right) \mathcal{V} y=\mu \mathcal{V}^{T} \mathbf{M} \mathcal{V} y
$$

The associated eigenvectors are $v=\mathcal{V} y, \bar{v}=\mathcal{V} \bar{y}$. To find the eigenvalue closest to zero for (1.4), a version of inverse iteration can be applied.

AlgORITHM 1 (inverse iteration for (1.4)).

1. Given $V_{1} \in \mathbb{R}^{n}$ with $\left\|V_{1}\right\|_{2}=1$ and $D_{1}=1$, let $Z_{1}=V_{1} D_{1} V_{1}^{T}$.

2. For $j=1,2, \ldots$

2.1. Compute the eigenvalue approximation ${ }^{1}$

$$
\lambda_{j}=-\frac{\operatorname{trace}\left(\widetilde{A}_{j}^{T} D_{j} \widetilde{M}_{j} D_{j}+\widetilde{M}_{j}^{T} D_{j} \widetilde{A}_{j} D_{j}\right)}{\operatorname{trace}\left(\widetilde{B}_{j}^{T} D_{j} \widetilde{M}_{j} D_{j}+\widetilde{M}_{j}^{T} D_{j} \widetilde{B}_{j} D_{j}\right)},
$$

where

$$
\widetilde{A}_{j}=V_{j}^{T} \mathbf{A} V_{j}, \quad \widetilde{B}_{j}=V_{j}^{T} \mathbf{B} V_{j}, \quad \widetilde{M}_{j}=V_{j}^{T} \mathbf{M} V_{j} .
$$

2.2. If $\left(\lambda_{j}, Z_{j}\right)$ is accurate enough, then stop.

2.3. Else, solve

$$
\mathbf{A} Y_{j} \mathbf{M}^{T}+\mathbf{M} Y_{j} \mathbf{A}^{T}=F_{j}
$$

in factored form $Y_{j}=V_{j+1} D_{j+1} V_{j+1}^{T}$, where $F_{j}=\mathbf{B} Z_{j} \mathbf{M}^{T}+\mathbf{M} Z_{j} \mathbf{B}^{T}$.

2.4. Normalize: $D_{j+1} \leftarrow D_{j+1} /\left\|D_{j+1}\right\|_{F}$. Let $Z_{j+1}=V_{j+1} D_{j+1} V_{j+1}^{T}$.

If $\mathbf{A}$ is nonsingular, then (2.6) is equivalent to the Lyapunov equation

$$
S Y_{j}+Y_{j} S^{T}=\mathbf{A}^{-1} F_{j} \mathbf{A}^{-T},
$$

where $S=\mathbf{A}^{-1} \mathbf{M}$. Let $\operatorname{rank}\left(Z_{j}\right)=k$; it is reasonable to assume that $k \ll n$ (see [18]). The right-hand side of (2.7) can be represented by its truncated eigenvalue decomposition

$$
\mathbf{A}^{-1} F_{j} \mathbf{A}^{-T}=P_{j} C_{j} P_{j}^{T}
$$

\footnotetext{
${ }^{1}$ The Rayleigh quotient (2.4) can be derived using a property of Kronecker products (see [16, Exercise 25, p. 252]).
}

Copyright $@$ by SIAM. Unauthorized reproduction of this article is prohibited. 
which has rank at most $2 k$ and is easy to compute. ${ }^{2}$ Since we assume that $\mathbf{M}$ is nonsingular and the point $\left(u_{0}, \alpha_{0}\right)$ is in the stable regime, all the eigenvalues of $S$ lie in the left half of the complex plane. This guarantees that (2.7) has a unique solution (see $[1$, Chapter 6]).

Theorem 2.2 implies that $Z_{c}$ has rank 2 , so when $Z_{j}$ has converged, the right-hand side of (2.7), namely (2.8), has rank 4 . For efficient computation of (2.7), we would like to work with $k=2$. However, in the first few iterations, when $Z_{j}$ has not converged yet, $k$ can be much larger than 2 (although $k \ll n$ ). A rank-reduction procedure is introduced in [18] to guarantee a small, fixed $k$. Before step 2.2 in Algorithm 2, we project the eigenvalue problem (1.4) onto the subspace $V_{j}$. This leads to the $k \times k$ eigenvalue problem

$$
\widetilde{M}_{j} \widetilde{Z}_{j} \widetilde{A}_{j}^{T}+\widetilde{A}_{j} \widetilde{Z}_{j} \widetilde{M}_{j}^{T}+\widetilde{\lambda}_{j}\left(\widetilde{M}_{j} \widetilde{Z}_{j} \widetilde{B}_{j}^{T}+\widetilde{B}_{j} \widetilde{Z}_{j} \widetilde{M}_{j}^{T}\right)=0
$$

where $\widetilde{A}_{j}, \widetilde{B}_{j}, \widetilde{M}_{j}$ are computed in (2.5). For $k \ll n,(2.9)$ can be solved using Algorithm 1 with a direct Lyapunov solver (see [3], [15]) in step 2.3. According to Theorem 2.2, $\widetilde{Z}_{j}$ has rank 2. Let the eigenvalue decomposition of $\widetilde{Z}_{j}$ be $\widetilde{V}_{j} \widetilde{D}_{j} \widetilde{V}_{j}^{T}$, where $\widetilde{V}_{j} \in \mathbb{R}^{k \times 2}$ and $\widetilde{D}_{j} \in \mathbb{R}^{2 \times 2}$. We update the eigenvector $Z_{j}=V_{j} D_{j} V_{j}^{T}$ by $\left(V_{j} \widetilde{V}_{j}\right) \widetilde{D}_{j}\left(V_{j} \widetilde{V}_{j}\right)^{T}$. The new eigenvector has rank 2 and it forces the residual of (1.4) to be orthogonal to $V_{j}$. With the rank-reduction procedure, the right-hand side of (2.7) will be of rank 2 in the first iteration and of rank 4 in all subsequent iterations, which is desirable for the Lyapunov solvers. The modified inverse iteration for (1.4) now reads as follows.

Algorithm 2 (inverse iteration for (1.4) with rank reduction).

1. Given $V_{1} \in \mathbb{R}^{n}$ with $\left\|V_{1}\right\|_{2}=1$ and $D_{1}=1$, let $Z_{1}=V_{1} D_{1} V_{1}^{T}$ and $k=1$.

2. For $j=1,2, \ldots$

2.1. Compute (2.5), and solve for the eigenvalue $\widetilde{\lambda}_{j}$ of (2.9) closest to zero and its eigenvector $\widetilde{Z}_{j}=\widetilde{V}_{j} \widetilde{D}_{j} \widetilde{V}_{j}^{T}$, where $\widetilde{V}_{j} \in \mathbb{R}^{k \times r}$ and $\widetilde{D}_{j} \in \mathbb{R}^{r \times r}$ with $r=1(j=1)$ or $2(j \geq 2)$.

2.2. Set $Z_{j}=\mathcal{V}_{j} \widetilde{D}_{j} \mathcal{V}_{j}^{T}$ and $\lambda_{j}=\widetilde{\lambda}_{j}$, where $\mathcal{V}_{j}=V_{j} \widetilde{V}_{j}$.

2.3. If $\left(\lambda_{j}, Z_{j}\right)$ is accurate enough, then stop.

2.4. Else, solve for $Y_{j}$ from

$$
S Y_{j}+Y_{j} S^{T}=P_{j} C_{j} P_{j}^{T}
$$

in factored form $Y_{j}=V_{j+1} D_{j+1} V_{j+1}^{T}$.

3. A block Krylov Lyapunov solver. In this section, we discuss the block Krylov method for solving the Lyapunov equation

$$
S Y+Y S^{T}=P C P^{T},
$$

where $S=\mathbf{A}^{-1} \mathbf{M} \in \mathbb{R}^{n \times n}, P \in \mathbb{R}^{n \times p}$ is orthonormal, and $C \in \mathbb{R}^{p \times p}$ is diagonal, with $p \ll n$. Let $K$ be a $k$-dimensional subspace of $\mathbb{R}^{n}$ and let $V$ be an orthonormal basis of $K$. Projection methods for (3.1) seek an approximate solution of the form $\widehat{Y}(Q)=V Q V^{T}$ with $Q \in \mathbb{R}^{k \times k}$ by imposing the so-called Galerkin condition; i.e., the residual $R(Q)=S \widehat{Y}(Q)+\widehat{Y}(Q) S^{T}-P C P^{T}$ of (3.1) must satisfy

\footnotetext{
${ }^{2}$ Let $T=\mathbf{A}^{-1} \mathbf{B}$; then $\mathbf{A}^{-1} F_{j} \mathbf{A}^{-T}=\left(\frac{\sqrt{2}}{2}\left[T V_{j}+S V_{j}, T V_{j}-S V_{j}\right]\right)\left[\begin{array}{ll}D_{j} & -D_{j}\end{array}\right]\left(\frac{\sqrt{2}}{2}\left[T V_{j}+S V_{j}\right.\right.$, $\left.\left.T V_{j}-S V_{j}\right]\right)^{T}$.
} 
$\langle Z, R(Q)\rangle=\operatorname{tr}\left(Z R(Q)^{T}\right)=0$ for any matrix $Z$ of the form $V \mathcal{G} V^{T}$ with $\mathcal{G} \in \mathbb{R}^{k \times k}$ (see [22]). The only $Q$ that satisfies this condition is the solution to the projected problem (see [22])

$$
\left(V^{T} S V\right) Q+Q\left(V^{T} S V\right)^{T}=\left(V^{T} P\right) C\left(V^{T} P\right)^{T} .
$$

In the block Krylov method (see [17], [22]), the subspace $K$ is chosen to be

$$
\mathcal{K}_{m}(S, P)=\operatorname{span}\left\{P, S P, S^{2} P, \ldots, S^{m-1} P\right\} .
$$

(The dimension of $\mathcal{K}_{m}(S, P)$ is $m p$.) One theoretical motivation for selecting such a subspace is that if all the eigenvalues of $S$ lie in the left half of the complex plane, then the analytic solution of (3.1) can be expressed as $-\int_{0}^{\infty} \exp (S t) P C P^{T} \exp \left(S^{T} t\right) d t$ (see $[1$, Chapter 6]). We use the block Arnoldi method to compute an orthonormal basis for $\mathcal{K}_{m}(S, P)$. Similar to the standard Arnoldi method, the block Arnoldi process computes a decomposition

$$
S V=V H_{m}+V_{m+1} H_{m+1, m} E_{m}^{T},
$$

where $V=\left[V_{1}, \ldots, V_{m}\right] \in \mathbb{R}^{n \times m p}$ is an orthonormal basis for $\mathcal{K}_{m}(S, P), H_{m} \in$ $\mathbb{R}^{m p \times m p}$ is a block upper-Hessenberg matrix with $p \times p$ blocks $H_{i, j}$, and $E_{m} \in \mathbb{R}^{m p \times p}$ consists of the last $p$ columns of the identity matrix of order $m p$. By the Arnoldi relationship (3.4), the projected problem (3.2) is

$$
H_{m} Q+Q H_{m}^{T}=\left[\begin{array}{ccc}
C & \cdots & 0 \\
\vdots & \ddots & \vdots \\
0 & \cdots & 0
\end{array}\right]=\widetilde{C}
$$

which, assuming $m p \ll n$, can be solved by direct methods. An algorithmic form of the block Krylov method for solving (3.1) is given below.

Algorithm 3 (the block Krylov method for (3.1)).

1. Given a tolerance $\tau$. Let $V_{1}=V=P$.

2. For $m=1,2, \ldots$

$$
\begin{aligned}
& \text { 2.1. } W=S V_{m} . \\
& \text { for } i=1, \ldots, m \\
& H_{i, m} \leftarrow V_{i}^{T} W \\
& W \leftarrow W-V_{i} H_{i, m} .
\end{aligned}
$$

2.2. Solve the smaller Lyapunov equation (3.5).

2.3. Compute the reduced QR factorization of $W: W=V_{m+1} H_{m+1, m}$.

2.4. Compute the residual norm $\|R(Q)\|_{F}$.

2.5. If $\|R(Q)\|_{F}<\tau$, then stop.

2.6. Else, $V \leftarrow\left[V, V_{m+1}\right]$.

We outline some of the computational issues associated with this algorithm. Since $S=\mathbf{A}^{-1} \mathbf{M}$, in step 2.1, we need to solve $p$ linear systems of the form

$$
\mathbf{A} x=\mathbf{M} y
$$

for $x$. Notice that we do not need to form the approximate solution $\widehat{Y}(Q)=V Q V^{T}$ explicitly. Instead, only the factors $V$ and $Q$ are stored. To compute the residual norm $\|R(Q)\|_{F}$, first notice that for any symmetric $Q$,

$$
R(Q)=\left[V, V_{m+1}\right]\left[\begin{array}{cc}
H_{m} Q+Q H_{m}^{T}-\widetilde{C} & Q E_{m} H_{m+1, m}^{T} \\
H_{m+1, m} E_{m}^{T} Q & 0
\end{array}\right]\left[V, V_{m+1}\right]^{T}
$$






(a) Decay of the eigenvalues of $Q$

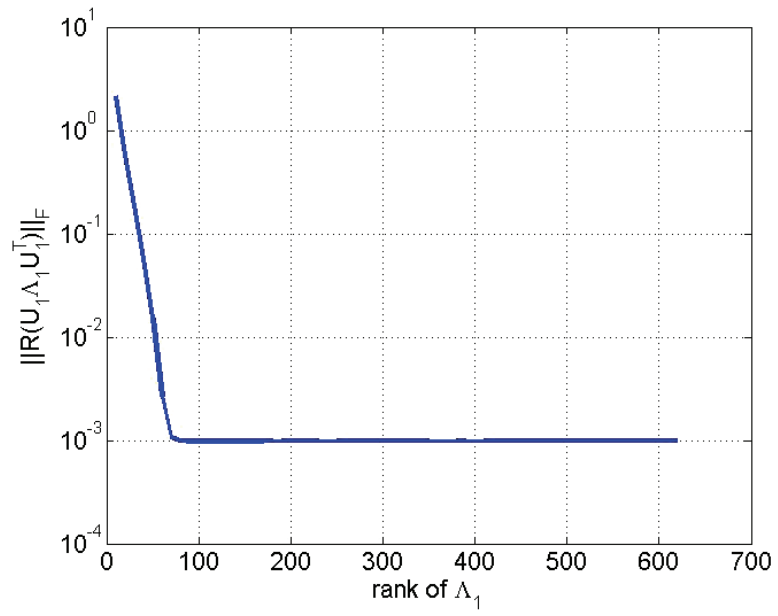

(b) Residual norm for different ranks of truncation

FIG. 3.1. Low-rank approximation of the solution to the Lyapunov equation $(n=37168)$.

(see [17]). By (3.5) and (3.7), $\|R(Q)\|_{F}=\sqrt{2}\left\|Q E_{m} H_{m+1, m}^{T}\right\|_{F}$, which is cheap to compute. Let $Q=U \Lambda U^{T}$ be the eigenvalue decomposition of $Q$, where $\Lambda=$ $\operatorname{diag}\left(\lambda_{1}, \lambda_{2}, \ldots, \lambda_{m}\right)$ holds the eigenvalues of $Q$, where the moduli are in decreasing order. The computed solution $\widehat{Y}(Q)$ can usually be truncated to a (much) lower rank without affecting the residual norm:

$$
\widehat{Y}(Q)=(V U) \Lambda(V U)^{T}=\left(V\left[U_{1}, U_{2}\right]\right)\left[\begin{array}{ll}
\Lambda_{1} & \\
& \Lambda_{2}
\end{array}\right]\left(V\left[U_{1}, U_{2}\right]\right)^{T} \approx \widehat{Y}\left(U_{1} \Lambda_{1} U_{1}^{T}\right) .
$$

In order to do this, we increase the rank of $\Lambda_{1}$ until the residual norm of the truncated solution, $\left\|R\left(U_{1} \Lambda_{1} U_{1}^{T}\right)\right\|_{F}$, is smaller than a prescribed tolerance $\tau$. For example, consider the Lyapunov equation arising from the first iteration of Algorithm 2 when applied to the flow over an obstacle (details of this example are given in section 5.2). Let the tolerance $\tau=10^{-3}$. The solution computed by Algorithm 3 has rank 628 and can be truncated to rank 80 without significantly affecting its accuracy. Figure 3.1(a)

Copyright $@$ by SIAM. Unauthorized reproduction of this article is prohibited. 
shows the decay of eigenvalues of $Q$, and Figure 3.1(b) depicts the residual norm of truncated solutions corresponding to various choices of $\Lambda_{1}$.

In our experiments, we have observed that when applying Algorithm 2 to problems arising from fluid mechanics, solving the Lyapunov equation (3.1) accurately can be quite expensive, especially at the early stages of the computation when the eigenvector $Z_{j}$ has not converged yet. In the next section, we will show that it is in fact not necessary to solve (3.1) accurately in the first few iterations of Algorithm 2.

Different choices of the subspace $K$ lead to variants of the standard Krylov method described here, for example, the extended Krylov subspace method [23] and the rational Krylov subspace method [8]. Some results for the alternative methods will be given in section 5.3.

Remark. Necessary and sufficient conditions for the projected Lyapunov equation (3.5) to have a unique solution is that $\theta_{i}+\theta_{j} \neq 0$, where $\left(\theta_{i}, \theta_{j}\right)$ is any pair of eigenvalues of $H_{m}$. To guarantee this, in the literature on projection methods for Lyapunov equations, it is common to require that the field of values of $S$ lie in one half of the complex plane (the imaginary axis not included). This is a rather strong condition which is not satisfied by matrices in our numerical experiments described in section 5 . (Instead, they satisfy only a weaker condition that all their eigenvalues lie in the left half of the complex plane.) Thus, it is possible that the solution to (3.5) does not exist or is not unique, which will lead to a breakdown of the projection method. We never encountered this difficulty in our experiments.

4. Inexact inverse iteration. In this section, we first review the main results from the previous work of Robbé, Sadkane, and Spence [19] on inexact inverse iteration, and based on their idea, we propose an inexact inverse iteration for solving the eigenvalue problem (1.4). Suppose that a cluster $(k \ll n)$ of eigenvalues of $A \in \mathbb{R}^{n \times n}$ near a shift $\sigma$ is wanted. The standard approach for this problem is inverse iteration, which requires the solution of $k$ linear systems

$$
(A-\sigma I) X_{i}=X_{i-1}
$$

at each step. Solving (4.1) exactly can be very challenging if $n$ is large, which is typical when $A$ arises from discretization of two- or three-dimensional PDEs. Therefore, the system (4.1) is often solved inexactly using iterative methods. This approach is referred to as an inner-outer iterative method: the inner iteration refers to the iterative solution of (4.1), and the outer iteration is inverse iteration for eigenvalues. For simplicity, let $k=1$ and $\sigma=0$; that is, suppose we are looking for the eigenvalue closest to zero. The inexact inverse iteration in this case is as follows.

Algorithm 4 (inexact inverse iteration).

1. Given a tolerance $\tau, \delta>0$ and the starting guess $z_{1}$ with $\left\|z_{1}\right\|_{2}=1$.

2. For $j=1,2, \ldots$

2.1. Compute the eigenvalue estimate: $\lambda_{j}=z_{j}^{T} A z_{j}$.

2.2. Set $\mathfrak{r}_{j}=A z_{j}-\lambda_{j} z_{j}$ and test convergence.

2.3. Solve $A y_{j}=z_{j}$ for $y_{j}$ inexactly such that $r_{j}=A y_{j}-z_{j}$ with

$$
\left\|r_{j}\right\|_{2}<\delta\left\|\mathfrak{r}_{j}\right\|_{2}
$$

2.4. Normalize: $z_{j+1}=y_{j} /\left\|y_{j}\right\|_{2}$.

Since $\delta$ is fixed, the stopping criterion (4.2) implies the following: at the early stage of the eigenvalue computation, when $\left\|\mathfrak{r}_{j}\right\|_{2}$ is still large, the inner iteration does not need to be very accurate either; as $\left(\lambda_{j}, z_{j}\right)$ converges to the true solution 
(i.e., $\left\|\mathfrak{r}_{j}\right\|_{2}$ gets smaller), (4.1) will be solved more and more accurately. It was shown in [19] that with this strategy, the number of inner iterations will not increase as the outer iteration proceeds.

We have a similar situation here: we want to compute the eigenvalue of (1.4) closest to zero using Algorithm 2, which requires the solution of (2.10) at each step. Note that $\|z\|_{2}=\|Z\|_{F}$ if $z=\operatorname{vec}(Z)$. Moreover, for $\mathbf{A}$ nonsingular, (1.4) is equivalent to

$$
S Z+Z S^{T}+\lambda\left(S Z T^{T}+T Z S^{T}\right)=0,
$$

where $S=\mathbf{A}^{-1} \mathbf{M}$ and $T=\mathbf{A}^{-1} \mathbf{B}$. Therefore, in Algorithm 2, the stopping criterion

$$
\left\|R_{j}\right\|_{F}<\delta\left\|\Re_{j}\right\|_{F}
$$

is used for the inner iteration (e.g., Algorithm 3) for (2.10), where $\mathfrak{R}_{j}=S Z_{j}+Z_{j} S^{T}+$ $\lambda_{j}\left(S Z_{j} T^{T}+T Z_{j} S^{T}\right)$ and $R_{j}=S Y_{j}+Y_{j} S^{T}-P_{j} C_{j} P_{j}^{T}$. Based on Algorithms 2 and 4 , we propose the following version of inexact inverse iteration for solving (1.4).

ALGORITHM 5 (inexact inverse iteration for (1.4) with rank reduction).

1. Given $V_{1} \in \mathbb{R}^{n}$ with $\left\|V_{1}\right\|_{2}=1$ and $D_{1}=1$, let $Z_{1}=V_{1} D_{1} V_{1}^{T}$ and $k=1$. Given $\delta>0$.

2. For $j=1,2, \ldots$

2.1. Compute (2.5), and solve for the eigenvalue $\widetilde{\lambda}_{j}$ of (2.9) closest to zero and its eigenvector $\widetilde{Z}_{j}=\widetilde{V}_{j} \widetilde{D}_{j} \widetilde{V}_{j}^{T}$, where $\widetilde{V}_{j} \in \mathbb{R}^{k \times r}$ and $\widetilde{D}_{j} \in \mathbb{R}^{r \times r}$ with $r=1(j=1)$ or $2(j \geq 2)$.

2.2. Set $Z_{j}=\mathcal{V}_{j} \widetilde{D}_{j} \mathcal{V}_{j}^{T}$ and $\lambda_{j}=\widetilde{\lambda}_{j}$, where $\mathcal{V}_{j}=V_{j} \widetilde{V}_{j}$.

2.3. Compute $\left\|\Re_{j}\right\|_{F}$ and test convergence.

2.4. Solve for $Y_{j}$ from $S Y_{j}+Y_{j} S^{T}=P_{j} C_{j} P_{j}^{T}$ in factored form $Y_{j}=$ $V_{j+1} D_{j+1} V_{j+1}^{T}$ such that $\left\|R_{j}\right\|_{F}<\delta\left\|\Re_{j}\right\|_{F}$.

2.5. Truncate the solution $Y_{j}$ to rank $k_{j}: V_{j+1} \leftarrow V_{j+1}\left(:, 1: k_{j}\right)$.

Remark. An alternative choice of the pair of residuals would be $\mathfrak{R}_{j}^{\prime}=\mathbf{M} Z_{j} \mathbf{A}^{T}+$ $\mathbf{A} Z_{j} \mathbf{M}^{T}+\lambda_{j}\left(\mathbf{M} Z_{j} \mathbf{B}^{T}+\mathbf{B} Z_{j} \mathbf{M}^{T}\right)$ and $R_{j}^{\prime}=\mathbf{A} Y_{j} \mathbf{M}^{T}+\mathbf{M} Y_{j} \mathbf{A}^{T}-F_{j}$, which are the residuals of (1.4) and (2.6), respectively. We prefer the choice used in Algorithm 5 because of cost considerations. $\left\|R_{j}\right\|_{F}$ is available at almost no cost due to (3.7), and since $Z_{j}=\mathcal{V}_{j} \widetilde{D}_{j} \mathcal{V}_{j}^{T}$ has rank two, $\Re_{j}$ has rank four and the dominant cost of computing $\left\|\mathfrak{R}_{j}\right\|_{F}$ is the solution of four systems with coefficient matrix $\mathbf{A}$, allowing us to compute $S \mathcal{V}_{j}$ and $T \mathcal{V}_{j} .{ }^{3}$ In contrast, although it is trivial to compute the Frobenius norm of the rank-four $\mathfrak{R}_{j}^{\prime}$, it can be very expensive to evaluate $\left\|R_{j}^{\prime}\right\|_{F}$ : by (3.7) and the relation $R_{j}^{\prime}=\mathbf{A} R_{j} \mathbf{A}^{T}$, computing $\left\|R_{j}^{\prime}\right\|_{F}$ at the $m$ th step of Algorithm 3 requires $p(m+1)$ matrix-vector products with $\mathbf{A}$, where $p$ is the rank of the right-hand side of (2.10). If a large number of Arnoldi steps is needed, which is indeed the case in our numerical experiments, then monitoring $\left\|R_{j}^{\prime}\right\|_{F}$ instead of $\left\|R_{j}\right\|_{F}$ will be much more expensive.

5. Numerical results. In this section, we apply Algorithm 5 to 2 twodimensional models of incompressible flows that lose stability because of Hopf bifurcation, namely, driven-cavity flow and flow over an obstacle. The numerical results support the theory of [18] and show that the algorithm we propose is robust.

In the previous sections, we always assumed that the mass matrix $\mathbf{M}$ is nonsingular. However, as given by (1.6), the mass matrix in our examples is singular. This

\footnotetext{
${ }^{3}$ In order to solve (2.10), A has been prefactored or a preconditioner for it has been computed.
} 
implies that (1.2) has an infinite eigenvalue (i.e., the eigenvalue that corresponds to the zero eigenvalue of $S$ ) of multiplicity $2 n_{p}$ (see [5]). As shown in [5], however, replacement of $\mathbf{M}$ with the nonsingular, shifted mass matrix

$$
\mathbf{M}_{\sigma}=\left[\begin{array}{cc}
-G & \sigma B^{T} \\
\sigma B & 0
\end{array}\right]
$$

maps the infinite eigenvalue of (1.2) to $\sigma^{-1}$ and leaves the finite ones unchanged. With a proper choice of $\sigma$, the rightmost eigenvalue(s) of (1.2) will not be changed, which means that stability analysis will not be affected. In our computations, we use the shifted mass matrix (5.1) with $\sigma=-10^{-2}$ instead of the $\mathbf{M}$ given in (1.6). The infinite eigenvalues of $(1.2)$ are mapped to $-10^{2}$, which is well away from its rightmost eigenvalues. In addition, the matrix $\mathbf{B}=\frac{d \mathcal{J}}{d \nu}\left(\nu_{0}\right)$ was approximated using a forward difference approximation.

All numerical results were obtained using MATLAB 7.8.0 (R2009a), on a PC with a $1.60 \mathrm{GHz}$ processor, and $4 \mathrm{~GB}$ of RAM.

5.1. Example 1: Driven-cavity flow. This is a classic test problem used in fluid dynamics, a model of the flow in a unit-square cavity with the lid moving from left to right. We use the software package IFISS (see [9]) to compute the steadystate solution of (1.5). The left plot of Figure 5.1 shows exponentially distributed streamlines of a steady solution. Studies of the critical Reynolds number $R e_{c}$ for this problem show it to be around 8000. (For example, the reported value is 7998.5 in [10], 7960 in [11], between 8017.6 and 8018.8 in [2], and between 8000 and 8050 within less than $1 \%$ error in [4].) The rightmost eigenvalues at the critical Reynolds number are also provided in [10] $( \pm \beta i \approx \pm 2.8356 i)$ and $[11]( \pm \beta i \approx \pm 2.837 i)$. The right plot of Figure 5.1 shows the eigenvalues of $\mathcal{J} x=\mu \mathbf{M} x$ at $R e=8076$, a value slightly larger than the critical value $R e_{c}$. As is clearly seen, there are many complex eigenvalues near the imaginary axis, and, in fact, it is a very difficult problem to find out precisely which eigenpair crosses the imaginary axis to cause the loss of stability.
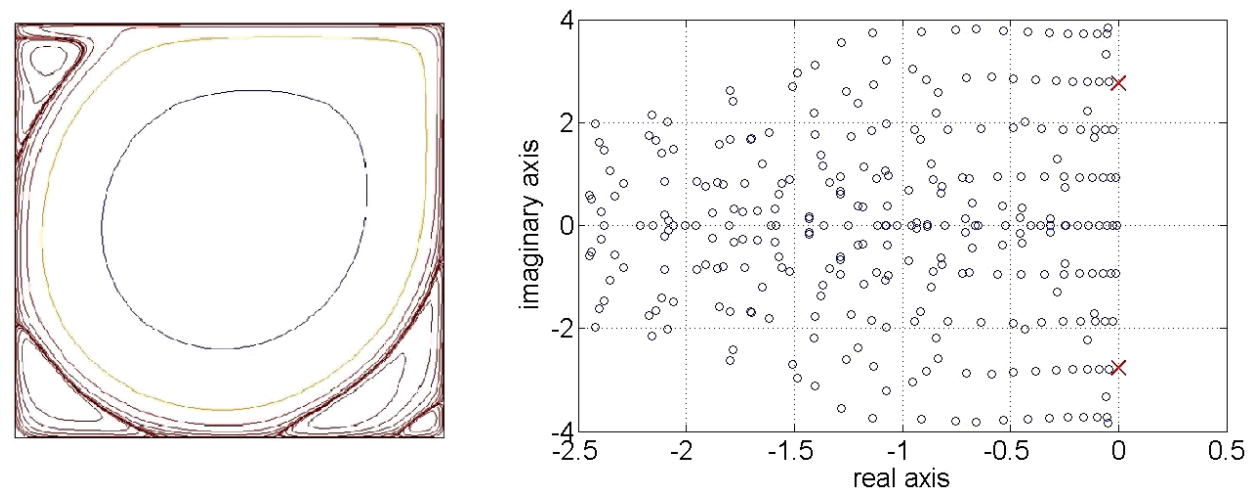

FIG. 5.1. Driven-cavity flow. Left plot: Exponentially distributed streamlines at Re $=7500$. Right plot: The 300 eigenvalues with smallest modulus of $\mathcal{J} x=\mu \mathbf{M} x$ computed by the implicitly restarted Arnoldi method at $R e=8076$ (the crosses denote the rightmost eigenvalues).

We use a $Q_{2}-Q_{1}$ mixed finite element discretization and three meshes: $64 \times 64$ $(n=9539), 128 \times 128(n=37507)$, and $256 \times 256(n=148739)$. Algorithm 5 is tested on the three problems arising from the three meshes of discretization, with tests for three choices $\delta=1,10^{-1}$, and $10^{-2}$ in (4.4). Let the Reynolds number at the starting 
TABLE 5.1

Driven-cavity flow $\left(256 \times 256\right.$ mesh, $\left.R e_{0}=7800\right)$ for $\delta=1,10^{-1}, 10^{-2}$ in (4.4).

\begin{tabular}{|c|c|c|c|c|c|c|c|}
\hline$j$ & $R e_{j}$ & $\mu_{j}$ & $\left\|r_{j}\right\|_{2}$ & $\left\|\Re_{j}\right\|_{F}$ & $\left\|R_{j}\right\|_{F}$ & $m_{j}$ & $k_{j}$ \\
\hline & & \multicolumn{6}{|c|}{$\delta=1$} \\
\hline 1 & -219 & $2.64515 \mathrm{e}-12$ & $1.29459 \mathrm{e}-01$ & $4.94209 \mathrm{e}+1$ & $4.86367 \mathrm{e}+1$ & 322 & 90 \\
\hline 2 & 8014 & $2.81408 \mathrm{i}$ & $1.72108 \mathrm{e}-06$ & $1.52388 \mathrm{e}-2$ & $1.48458 \mathrm{e}-2$ & 424 & 160 \\
\hline 3 & 8080 & $2.80919 \mathrm{i}$ & $7.33553 \mathrm{e}-08$ & $4.42196 \mathrm{e}-4$ & $3.87001 \mathrm{e}-4$ & 444 & 160 \\
\hline 4 & 8077 & $2.80960 \mathrm{i}$ & $3.43710 \mathrm{e}-09$ & $1.61958 \mathrm{e}-5$ & $1.58346 \mathrm{e}-5$ & 448 & 170 \\
\hline \multirow[t]{3}{*}{5} & 8077 & $2.80960 \mathrm{i}$ & $1.04455 \mathrm{e}-10$ & $5.90803 \mathrm{e}-7$ & - & - & - \\
\hline & & & & & Total: & 1638 & \\
\hline & & \multicolumn{6}{|c|}{$\delta=10^{-1}$} \\
\hline 1 & -219 & $2.64515 \mathrm{e}-12$ & $1.29459 \mathrm{e}-01$ & $4.94209 \mathrm{e}+1$ & $4.79199 \mathrm{e}+0$ & 510 & 120 \\
\hline 2 & 8173 & $2.81562 \mathrm{i}$ & $2.54877 \mathrm{e}-06$ & $2.57187 \mathrm{e}-2$ & $2.43960 \mathrm{e}-3$ & 536 & 190 \\
\hline 3 & 8083 & $2.80915 \mathrm{i}$ & $7.32265 \mathrm{e}-08$ & $3.57523 \mathrm{e}-4$ & $3.37686 \mathrm{e}-5$ & 552 & 210 \\
\hline 4 & 8077 & $2.80960 \mathrm{i}$ & $4.06199 \mathrm{e}-09$ & $2.07058 \mathrm{e}-5$ & $2.00807 \mathrm{e}-6$ & 532 & 210 \\
\hline \multirow[t]{3}{*}{5} & 8077 & $2.80960 \mathrm{i}$ & $1.53027 \mathrm{e}-10$ & $8.23020 \mathrm{e}-7$ & - & - & - \\
\hline & & & & & Total: & 2130 & \\
\hline & & \multicolumn{6}{|c|}{$\delta=10^{-2}$} \\
\hline 1 & -219 & $2.64515 \mathrm{e}-12$ & $1.29459 \mathrm{e}-01$ & $4.94209 \mathrm{e}+1$ & $4.88503 \mathrm{e}-1$ & 914 & 190 \\
\hline 2 & 8291 & $2.81758 \mathrm{i}$ & $2.85886 \mathrm{e}-06$ & $3.19166 \mathrm{e}-2$ & $3.14783 \mathrm{e}-4$ & 724 & 260 \\
\hline 3 & 8082 & $2.80908 \mathrm{i}$ & $7.05097 \mathrm{e}-08$ & $4.49294 \mathrm{e}-4$ & $4.32428 \mathrm{e}-6$ & 728 & 270 \\
\hline 4 & 8077 & $2.80960 \mathrm{i}$ & $5.23912 \mathrm{e}-09$ & $1.97292 \mathrm{e}-5$ & $1.92897 \mathrm{e}-7$ & 724 & 260 \\
\hline \multirow[t]{2}{*}{5} & 8077 & $2.80960 \mathrm{i}$ & $1.51600 \mathrm{e}-10$ & $6.70318 \mathrm{e}-7$ & - & - & - \\
\hline & & & & & Total: & 3090 & \\
\hline
\end{tabular}

point, $R e_{0}$, be about 250 smaller than its critical value, $R e_{c}$. The goal of our tests is to find out whether Algorithm 5 is able to approximate the difference $\lambda_{c}$ between the two viscosities $\nu_{0}=\frac{1}{R e_{0}}$ and $\nu_{c}=\frac{1}{R e_{c}}$ and, in turn, give us a good estimate of $R e_{c}{ }^{4}$ The computational results for the finest mesh are reported in Table 5.1. $R e_{j}$ denotes the estimated value of $R e_{c}, \mu_{j}$ denotes the estimated $\beta i, r_{j}=\left(\mathbf{A}+\lambda_{j} \mathbf{B}\right) x_{j}-\mu_{j} \mathbf{M} x_{j}$ is the residual of (1.3), and $R_{j}, \mathfrak{R}_{j}$ are defined in the previous section. In addition, $m_{j}$ is the rank of the solution of (2.10) before truncation, and $k_{j}$ is the rank after truncation. The main cost of each iteration is the $m_{j}$ solves of linear systems with coefficient matrix A. The computation terminates when $\left\|r_{j}\right\|_{2}<10^{-9}$ is satisfied. In the first iteration, when a real, symmetric, and rank-one matrix $v v^{T}(v$ is a random vector in $\mathbb{R}^{n}$ ) is used as the eigenvector estimate of (1.4), the eigenvalue estimate $\lambda_{j}$ is quite far away from its true value $\lambda_{c}$, causing the estimated critical Reynolds number to be nonphysical (-219). However, starting from the second iteration, $\lambda_{j}$ converges rapidly to its true value. A fairly large Krylov subspace is needed to solve the Lyapunov equations, even when the tolerance is quite mild $\left(\left\|R_{j}\right\|_{F}<\left\|\Re_{j}\right\|_{F}\right)$. Computational results for the two coarser meshes can be found in Appendix A, and the same trend can be observed there.

As observed above, a commonly used method to locate the first Hopf point is to compute the rightmost eigenvalues of $\mathcal{J} x=\mu \mathbf{M} x$ for a set of points with increasing Reynolds numbers on the solution path $\mathcal{S}$, until a critical value is reached at which the real part of the rightmost eigenvalues becomes positive. We follow this approach to verify the results given by Algorithm 5. The details are as follows: for each point in the set, we compute the 250 eigenvalues with smallest modulus for $\mathcal{J} x=\mu \mathbf{M} x$ using MATLAB function "eigs" (with other parameters set to default values), which implements the implicitly restarted Arnoldi (IRA) method [24]. For the finest mesh,

\footnotetext{
${ }^{4}$ Let $\lambda_{c}=\nu_{c}-\nu_{0} ;$ then once $\lambda_{c}$ is approximated by Algorithm $5, R e_{c}$ can be estimated by $\frac{1}{\nu_{0}+\lambda_{c}}$.
} 
the critical Reynolds number found by this method is between 8075 and 8076 , and the rightmost eigenvalues are $\pm \beta i \approx \pm 2.80905 i$. This shows that Algorithm 5 yields good estimates of $R e_{c}$ and $\beta i$. The number 250 was obtained by trial and error. When only 200 eigenvalues with smallest modulus were computed, we could not find the rightmost eigenvalues.

Remark. Our goal is to have a robust method that detects instability without computing many eigenvalues, since we do not know in general how many eigenvalues need be computed to ensure that the rightmost ones have been found. It is also not straightforward to evaluate the cost of the IRA method when it is used to generate a set of eigenvalues in this way, because this cost is highly dependent on how various parameters are chosen. Consequently, we do not make a detailed cost comparison of the two methods. For the particular choice of parameters we made, i.e., computing the 250 eigenvalues with smallest modulus using "eigs" with default setting, at each Reynolds number in the set, the eigenvalue computation requires the solution of at least 500 linear systems with coefficient matrix $\mathcal{J}$, and typically many more. In our experience, locating $R e_{c}$ by monitoring the rightmost eigenvalues along $\mathcal{S}$ is much more expensive than Algorithm 5 with $\delta=1$.

5.2. Example 2: Flow over an obstacle. This example represents flow in a channel (dimension: $2 \times 8$ ) with a square obstacle (dimension: $0.5 \times 0.5$ ) in it. (In this case, the Reynolds number is defined to be $\frac{2}{\nu}$.) A Poiseuille flow profile is imposed on the inflow boundary, and a no-flow (zero velocity) condition is imposed on the walls. A Neumann condition is applied at the outflow boundary and automatically sets the mean outflow pressure to zero (see [9] for details). Again we use IFISS to compute the steady-state solution. Uniformly distributed streamlines of the steady solution are plotted in Figure 5.2(a). As in the previous example, we use $Q_{2}-Q_{1}$ mixed finite element discretization and apply Algorithm 5 (with $\delta=1,10^{-1}, 10^{-2}$ ) on three meshes: $32 \times 128(n=9512), 64 \times 256(n=37168)$, and $128 \times 512(n=146912)$.

We choose $R e_{0}$ to be 50 smaller than the critical value $R e_{c}$. The computational results for the finest mesh are reported in Table 5.2. Results given by the IRA method (see Example 1) are the following: $372 \leq R e_{c} \leq 373$ and $\pm \beta i \approx \pm 2.26578 i$. The 300 eigenvalues with smallest modulus at a Reynolds number slightly larger than $R e_{c}$ are plotted in Figure 5.2(b). As in the previous example, our algorithm gives good estimates of $R e_{c}$ and $\beta i$. This problem has significantly fewer eigenvalues near the imaginary axis, and the Krylov subspaces needed for the Lyapunov solves are also significantly smaller than for the cavity problem. Computational results for the other two meshes can be found in Appendix B.

Remark. Note that $\mathbf{A}+\lambda_{c} \mathbf{B}$ is only a linear approximation of the true Jacobian matrix $\mathcal{J}\left(\alpha_{c}\right)$. Therefore, the true eigenvalue of (1.4), (2.2), and (4.3) with smallest modulus is $\lambda_{c}+\epsilon$, where $\epsilon$ is a small error. The size of this error, $|\epsilon|$, depends on the distance between the chosen starting point $\left(u_{0}, \alpha_{0}\right)$ and the critical point $\left(u_{c}, \alpha_{c}\right)$ : the closer $\left(u_{c}, \alpha_{c}\right)$ is to $\left(u_{0}, \alpha_{0}\right)$, the better $\mathbf{A}+\lambda_{c} \mathbf{B}$ approximates $\mathcal{J}\left(\alpha_{c}\right)$ and the smaller $|\epsilon|$ gets.

5.3. Discussion of Lyapunov solvers. As observed above, the efficiency of Algorithm 5 depends largely on the cost of solving the large-scale Lyapunov equation (3.1) at each iteration. In section 3, we discussed the Krylov method which searches for an approximate solution $V Q V^{T}$, where $V$ is an orthonormal basis of the Krylov subspace $\mathcal{K}_{m}(S, P)=\operatorname{span}\left\{P, S P, S^{2} P, \ldots, S^{m-1} P\right\}$ and $Q$ solves the small projected problem obtained by imposing the Galerkin condition. As shown in Example 1 (driven-cavity flow), a large Krylov subspace is needed for this method to 


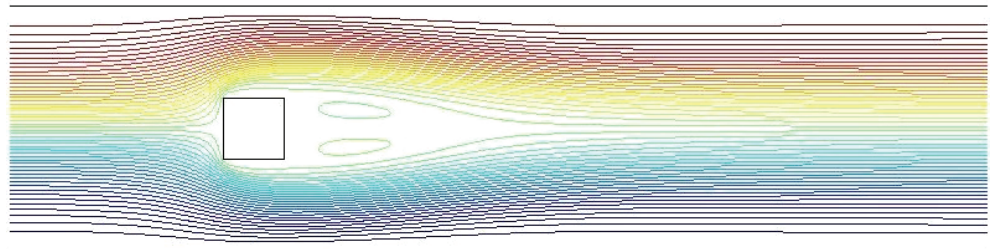

(a) Uniformly distributed streamlines at $R e=350$

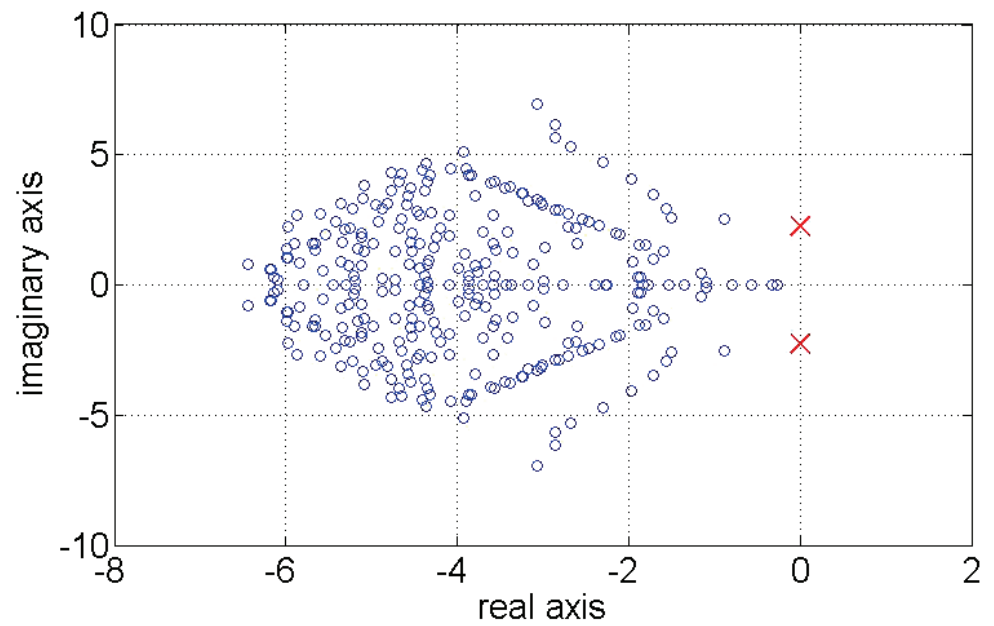

(b) The 300 eigenvalues with smallest modulus of $\mathcal{J} x=\mu \mathbf{M} x$ computed by the IRA method at $R e=373$ (the crosses denote the rightmost eigenvalues)

FiG. 5.2. Flow over an obstacle.

compute an accurate enough solution even for the mild tolerance $\left\|R_{j}\right\|_{F}<\left\|\Re_{j}\right\|_{F}$. This deficiency leads us to the exploration of alternative Lyapunov solvers.

A recently developed projection method is the rational Krylov subspace method (RKSM) [8]. Like the standard Krylov method, it projects a large Lyapunov equation onto a much smaller subspace, solves the small Lyapunov equation obtained by imposing the Galerkin condition, and projects the solution back to the original space. In this method, the Krylov subspace is defined to be

$$
\mathcal{K}_{m}(S, P, \mathbf{s})=\operatorname{span}\left\{P,\left(S-s_{1} I\right)^{-1} P, \ldots, \prod_{j=1}^{m-1}\left(S-s_{m-j} I\right)^{-1} P\right\},
$$

where $\mathbf{s}=\left[s_{1}, s_{2}, \ldots, s_{m-1}\right]^{T} \in \mathbb{C}^{m-1}$ is a vector of shifts that can be selected a priori or generated adaptively during computation. An algorithm that computes a decomposition similar to (3.4) for $\mathcal{K}_{m}(S, P, \mathbf{s})$ can be found in [21]. The use of such a subspace is first introduced by Ruhe for eigenvalue computation [20], where the shifts are placed around the target eigenvalues. In [7], RKSM is used to approximate $u(t)=\exp (S t) P \in \mathbb{R}^{n}$, where $S \in \mathbb{R}^{n \times n}$ is symmetric negative definite. An adaptive approach of choosing the shifts is proposed in [7] with the goal of minimizing the upper bound of the $L_{2}(0, \infty)$ error of the RKSM solution. This upper bound suggests that the shifts should lie on the imaginary axis, although it is shown in [7] that they can be restricted to the interval $\left[-\lambda_{\max },-\lambda_{\min }\right]$ on the real line, where $\lambda_{\max }$ and 
TABLE 5.2

Flow over an obstacle $\left(128 \times 512\right.$ mesh, $\left.R e_{0}=320\right)$ for $\delta=1,10^{-1}, 10^{-2}$ in (4.4).

\begin{tabular}{|c|c|c|c|c|c|c|c|}
\hline$j$ & $R e_{j}$ & $\mu_{j}$ & $\left\|r_{j}\right\|_{2}$ & $\left\|\Re_{j}\right\|_{F}$ & $\left\|R_{j}\right\|_{F}$ & $m_{j}$ & $k_{j}$ \\
\hline & & \multicolumn{6}{|c|}{$\delta=1$} \\
\hline 1 & -331 & $-6.21192 \mathrm{e}-13$ & $1.52776 \mathrm{e}-01$ & $4.38125 \mathrm{e}+0$ & $3.52097 \mathrm{e}+0$ & 68 & 10 \\
\hline 2 & 311 & $2.26820 \mathrm{i}$ & $2.28878 \mathrm{e}-04$ & $1.79583 \mathrm{e}-1$ & $1.77367 \mathrm{e}-1$ & 56 & 20 \\
\hline 3 & 378 & $2.27689 \mathrm{i}$ & $4.11801 \mathrm{e}-05$ & $5.72823 \mathrm{e}-3$ & $4.23584 \mathrm{e}-3$ & 68 & 20 \\
\hline 4 & 375 & $2.26633 \mathrm{i}$ & $9.82413 \mathrm{e}-06$ & $1.20449 \mathrm{e}-3$ & $6.69375 \mathrm{e}-4$ & 68 & 20 \\
\hline 5 & 373 & $2.26632 \mathrm{i}$ & $1.79954 \mathrm{e}-06$ & $2.34683 \mathrm{e}-4$ & $2.09699 \mathrm{e}-4$ & 64 & 30 \\
\hline 6 & 373 & $2.26661 \mathrm{i}$ & $2.42540 \mathrm{e}-07$ & $2.87217 \mathrm{e}-5$ & $2.67124 \mathrm{e}-5$ & 64 & 20 \\
\hline 7 & 373 & $2.26656 \mathrm{i}$ & $4.05258 \mathrm{e}-08$ & $5.38433 \mathrm{e}-6$ & $4.10305 \mathrm{e}-6$ & 64 & 20 \\
\hline 8 & 373 & $2.26656 \mathrm{i}$ & $5.45124 \mathrm{e}-09$ & $7.12393 \mathrm{e}-7$ & $4.27719 \mathrm{e}-7$ & 68 & 20 \\
\hline 9 & 373 & $2.26656 \mathrm{i}$ & $1.32615 \mathrm{e}-09$ & $1.82166 \mathrm{e}-7$ & $9.15168 \mathrm{e}-8$ & 68 & 20 \\
\hline \multirow[t]{3}{*}{10} & 373 & $2.26656 \mathrm{i}$ & $3.22020 \mathrm{e}-10$ & $3.99332 \mathrm{e}-8$ & - & - & - \\
\hline & & & & & Total: & 588 & \\
\hline & & \multicolumn{6}{|c|}{$\delta=10^{-1}$} \\
\hline 1 & -331 & $-6.21192 \mathrm{e}-13$ & $1.52776 \mathrm{e}-01$ & $4.38125 \mathrm{e}+0$ & $4.22028 \mathrm{e}-1$ & 202 & 30 \\
\hline 2 & 366 & $2.21977 \mathrm{i}$ & $1.44453 \mathrm{e}-04$ & $3.67019 \mathrm{e}-2$ & $3.34052 \mathrm{e}-3$ & 84 & 40 \\
\hline 3 & 368 & $2.26650 \mathrm{i}$ & $2.91683 \mathrm{e}-05$ & $3.77305 \mathrm{e}-3$ & $2.59429 \mathrm{e}-4$ & 80 & 30 \\
\hline 4 & 374 & $2.26727 \mathrm{i}$ & $3.07688 \mathrm{e}-06$ & $5.16995 \mathrm{e}-4$ & $3.16904 \mathrm{e}-5$ & 80 & 30 \\
\hline 5 & 373 & $2.26650 \mathrm{i}$ & $4.51259 \mathrm{e}-07$ & $5.51444 \mathrm{e}-5$ & $5.21710 \mathrm{e}-6$ & 76 & 40 \\
\hline 6 & 373 & $2.26657 \mathrm{i}$ & $4.14640 \mathrm{e}-08$ & $5.33721 \mathrm{e}-6$ & $4.04173 \mathrm{e}-7$ & 76 & 40 \\
\hline 7 & 373 & $2.26656 \mathrm{i}$ & $4.67350 \mathrm{e}-09$ & $6.55972 \mathrm{e}-7$ & $4.42397 \mathrm{e}-8$ & 80 & 40 \\
\hline \multirow[t]{3}{*}{8} & 373 & $2.26656 \mathrm{i}$ & $6.61702 \mathrm{e}-10$ & $9.81259 \mathrm{e}-8$ & - & - & - \\
\hline & & & & & Total: & 678 & \\
\hline & & \multicolumn{6}{|c|}{$\delta=10^{-2}$} \\
\hline 1 & -331 & $-6.21192 \mathrm{e}-13$ & $1.52776 \mathrm{e}-01$ & $4.38125 \mathrm{e}+0$ & $3.87099 \mathrm{e}-2$ & 254 & 40 \\
\hline 2 & 364 & $2.22687 \mathrm{i}$ & $5.37359 \mathrm{e}-04$ & $1.51434 \mathrm{e}-2$ & $1.36842 \mathrm{e}-4$ & 164 & 60 \\
\hline 3 & 370 & $2.26840 \mathrm{i}$ & $1.79202 \mathrm{e}-05$ & $2.39139 \mathrm{e}-3$ & $2.32630 \mathrm{e}-5$ & 156 & 60 \\
\hline 4 & 374 & $2.26678 \mathrm{i}$ & $2.40915 \mathrm{e}-06$ & $3.15403 \mathrm{e}-4$ & $2.86933 \mathrm{e}-6$ & 148 & 50 \\
\hline 5 & 373 & $2.26653 \mathrm{i}$ & $4.22143 \mathrm{e}-07$ & $5.32762 \mathrm{e}-5$ & $5.14646 \mathrm{e}-7$ & 132 & 50 \\
\hline 6 & 373 & $2.26657 \mathrm{i}$ & $9.53373 \mathrm{e}-09$ & $2.13741 \mathrm{e}-6$ & $1.82336 \mathrm{e}-8$ & 160 & 50 \\
\hline 7 & 373 & $2.26656 \mathrm{i}$ & $3.55204 \mathrm{e}-09$ & $6.02453 \mathrm{e}-7$ & $4.99433 \mathrm{e}-9$ & 160 & 50 \\
\hline \multirow[t]{2}{*}{8} & 373 & $2.26656 \mathrm{i}$ & $3.66251 \mathrm{e}-10$ & $5.45473 \mathrm{e}-8$ & - & - & - \\
\hline & & & & & Total: & 1174 & \\
\hline
\end{tabular}

$\lambda_{\min }$ are the largest and smallest eigenvalues of $S$, respectively. We present their formula for computing the next shift $s_{m}(m \geq 2)$ without going into detail:

$$
s_{m}=\arg \left(\max _{s \in \mathcal{I}} \frac{1}{\left|r_{m}(s)\right|}\right), \quad r_{m}(z)=\frac{\prod_{j=1}^{m}\left(z-\lambda_{j}^{(m)}\right)}{\prod_{j=1}^{m-1}\left(z-s_{j}\right)^{\frac{m}{m-1}}},
$$

where $\left\{\lambda_{j}^{(m)}\right\}_{j=1}^{m}$ are the Ritz values of $S$ on the Krylov subspace $\mathcal{K}_{m}=(S, P, \mathbf{s})$, $\left\{s_{j}\right\}_{j=1}^{m-1}$ are the shifts of previous iterations, and $\mathcal{I}=\left[-\lambda_{\max },-\lambda_{\min }\right]$. In each Arnoldi step, a new pole will be added to the denominator of $r_{m}(z)$ and the numerator of $r_{m}(z)$ will be completely changed. To start the computation, the first shift $s_{1}$ is set to be an estimate of $-\lambda_{\max }$ or $-\lambda_{\min }$, which must be provided by some means. In [8], it is shown that this adaptive computation of the shifts can be used to generate an efficient Krylov subspace for solving the Lyapunov equation (3.1). This is motivated by the relation between $\exp (S t) P$ and the analytic solution $\int_{0}^{\infty} \exp (S t) P C P^{T} \exp \left(S^{T} t\right) d t$ to (3.1). To generate the adaptive approach to a nonsymmetric $S$, [8] suggests replacing $\mathcal{I}=\left[-\lambda_{\max },-\lambda_{\min }\right]$ by $\mathcal{I}=\left[-R_{\max }(\lambda)\right.$, $\left.-\operatorname{Re}_{\min }(\lambda)\right]$. As before, $\operatorname{Re}_{\max }(\lambda)$ and $\operatorname{Re}_{\min }(\lambda)$ must be estimated beforehand (see [8] for a discussion). A convergence analysis of RKSM is given in [6]. 
Recall that in our problem, $S=\mathbf{A}^{-1} \mathbf{M}$, where $\mathbf{A} \in \mathbb{R}^{n \times n}$ is the Jacobian matrix and $\mathbf{M} \in \mathbb{R}^{n \times n}$ is the nonsingular, shifted mass matrix given by (5.1). Both $\mathbf{A}$ and $\mathbf{M}$ are large and sparse, and $\mathbf{A}$ is nonsymmetric. Each iteration of the standard Krylov method applied to (3.1) requires $p$ solves with the coefficient matrix $\mathbf{A}$ to compute the new Arnoldi block $V_{m+1}$, where $p$ is the rank of the right-hand side of (3.1). On the other hand, each iteration of RKSM requires $p$ solves with the coefficient matrices $\mathbf{M}-s_{j} \mathbf{A}$ to compute the new Arnoldi block $V_{m+1}$, and an extra $p$ solves with the coefficient matrix $\mathbf{A}$ to compute $S V_{m+1}$, which in turn gives us the Rayleigh quotient (see Algorithm 1 and Proposition 4.1 from [8]). While we can prefactor $\mathbf{A}$ or precompute a preconditioner for $\mathbf{A}$, we cannot do the same thing for $\mathbf{M}-s_{j} \mathbf{A}$, since the shift $s_{j}$ is different from iteration to iteration. Therefore, when Krylov subspaces of the same dimension are used to approximate the solution to (3.1), RKSM will be more expensive than the standard Krylov method. RKSM is competitive only when it can generate the solution with a smaller subspace.

The examples we consider in this discussion of Lyapunov solvers are the Lyapunov equations

$$
S Y_{j}+Y_{j} S^{T}=P_{j} C_{j} P_{j}^{T}, \quad j=1,2,3,4,
$$

arising from the first three iterations of Algorithm 5 (with $\delta=1$ and the standard Krylov Lyapunov solver) for driven-cavity flow on the two coarser meshes. The rank of the right-hand side is 2 for $j=1$ and 4 for $j=2,3,4$. The matrix $\mathbf{A}$ is prefactored. Let the residual of (5.4) be $R_{j}$. To compare the performance of the standard Krylov method and RKSM for solving (5.4), we have carried out the following numerical experiments.

We first compare the performance of the two methods. In Figure 5.3, we plot the decay of residual norm $\left(\left\|R_{j}\right\|_{F}\right)$ for both the standard Krylov method and RKSM as the dimension of the Krylov subspace $\mathcal{K}$ (see (3.3) and (5.2) for definitions) increases to an allowed maximum of 800 . In all four cases, RKSM has a faster asymptotic convergence rate than the standard Krylov method, and RKSM is able to find a much more accurate solution. For example, when $j=1$, the final residual norm of the RKSM solution is about $10^{-7}$, whereas that of the Krylov solution is only about $10^{-1}$.

Second, we compare the CPU times of the standard Krylov method and RKSM as well as the ranks of the solutions produced by them when the stopping criterion is $\left\|R_{j}\right\|_{F}<10^{-3}$. These results are reported in Table 5.3. Consider first the case $j=1$, where we performed the computation for two different mesh sizes. On both meshes, RKSM yields solutions with much lower rank, on the order of $24 \%$ to $35 \%$ of the rank of the Krylov solutions. It is also much cheaper than the standard Krylov method in terms of CPU time; for example, on the coarsest mesh, it takes RKSM 8 minutes to compute the solution with rank 426 but 63 minutes for the standard Krylov method to compute the solution of rank 1218. The high cost of RKSM per iteration is fully compensated for by its early convergence. In addition, when the mesh is refined, the rank of the RKSM solution seems to be mesh-independent (426 and 428), whereas the rank of the standard Krylov method increases noticeably (1218 and 1748). This suggests that the finer the mesh is, the more efficient RKSM will be compared with the Krylov method.

Next, consider analogous results for $j=2,3,4$, i.e., as the outer iteration proceeds. It can be seen from Figure 5.3 that for both Lyapunov solvers the Lyapunov equation becomes progressively easier to solve as $j$ increases. This is due to the fact 


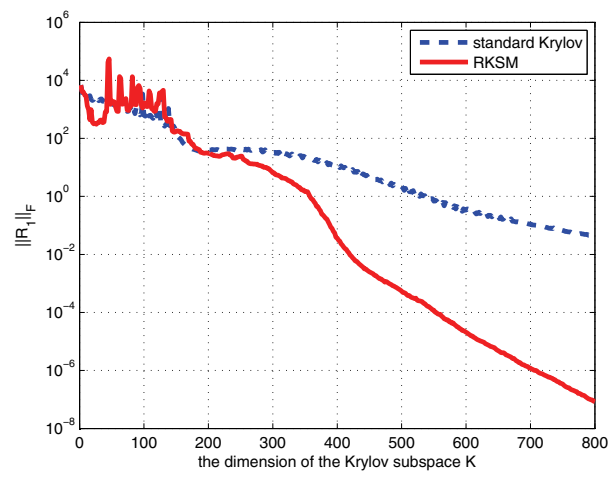

(a) $j=1$

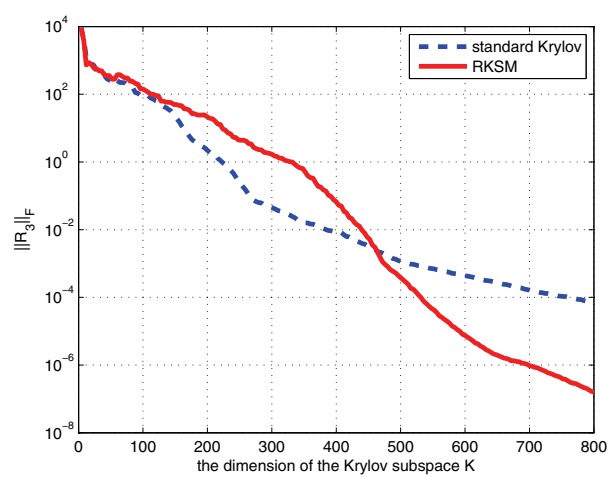

(c) $j=3$

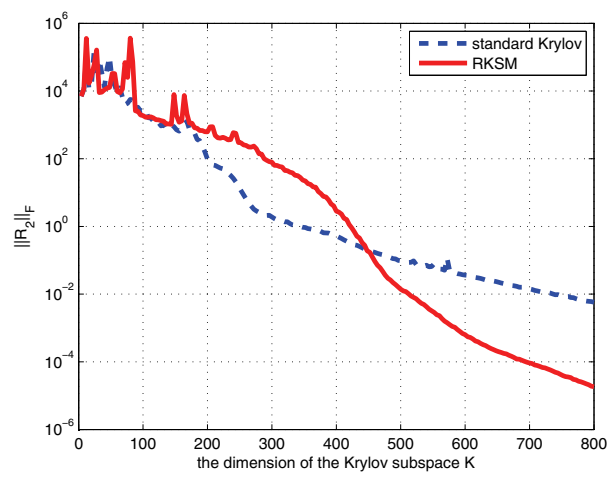

(b) $j=2$



(d) $j=4$

FIG. 5.3. Residual norm decay of the standard Krylov method and RKSM $(64 \times 64$ mesh).

TABLE 5.3

Rank of the approximate solution and CPU time.

\begin{tabular}{|c|c|c|c|c|c|c|c|c|}
\hline & \multicolumn{4}{|c|}{$64 \times 64$ mesh } & \multicolumn{4}{|c|}{$128 \times 128 \mathrm{mesh}$} \\
\hline$j$ & \multicolumn{2}{|c|}{ Krylov } & \multicolumn{2}{|c|}{ RKSM } & \multicolumn{2}{|c|}{ Krylov } & \multicolumn{2}{|c|}{ RKSM } \\
\hline 1 & 1218 & (63 min.) & & $(8$ min. $)$ & 1748 & (276 min.) & 428 & $(34$ min. $)$ \\
\hline 2 & 1024 & (15 min.) & 58 & $(10 \mathrm{~min})$. & & & & \\
\hline 3 & 516 & (1 min.) & 47 & $(6$ min. $)$ & & & & \\
\hline 4 & 312 & $(0.3$ min. $)$ & 428 & (5 min.) & & & & \\
\hline
\end{tabular}

that as $j$ increases, the starting block of both methods, $P_{j}$, gets "closer" to the eigenvectors associated with the dominant eigenvalues of the solution $Y_{j}$, and moreover, these dominant eigenvalues become more separate from the other eigenvalues of $Y_{j}$. Evidence for this is as follows. From section 2, we know that (normalized) $Y_{j}$ converges to the eigenvector $Z_{c}=\mathcal{V} \mathcal{D} \mathcal{V}^{T}$ of (1.4), where $\mathcal{V} \in \mathbb{R}^{n \times 2}$. Figure 5.4 shows the moduli of the 50 eigenvalues of $Y_{j}(j=1,2,3,4)$ with largest modulus. (The 800-dimensional RKSM solutions are taken as the exact $Y_{j}$ 's.) It is not difficult to see that as $j$ increases, the two eigenvalues of $Y_{j}$ with largest modulus become more 


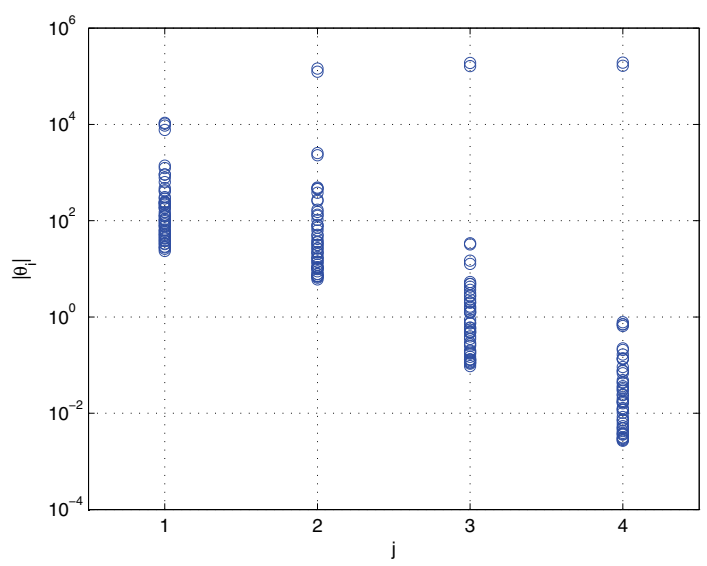

FIG. 5.4. Moduli of the 50 eigenvalues of $Y_{j}$ with largest modulus $(64 \times 64$ mesh $)$.

dominant. Let $U_{j} \in \mathbb{R}^{n \times 2}$ hold the eigenvectors of $Y_{j}$ associated with the two dominant eigenvalues, and let $\angle\left(P_{j}, U_{j}\right)$ denote the angle between the subspaces spanned by $P_{j}$ and $U_{j}$ (see [25] for the definition of the angle between two subspaces). The smaller this angle is, the closer the two subspaces are to being linearly dependent. We compute $\angle\left(P_{j}, U_{j}\right)$ for $j=1,2,3,4$ using MATLAB function "subspace" and obtain the following results: $\angle\left(P_{1}, U_{1}\right) \approx 1.5326, \angle\left(P_{2}, U_{2}\right) \approx 0.3564, \angle\left(P_{3}, U_{3}\right) \approx 0.0071$, and $\angle\left(P_{4}, U_{4}\right) \approx 0.0002$. Thus, $\angle\left(P_{j}, U_{j}\right)$ goes to zero rapidly as the outer iteration proceeds. Table 5.3 also suggests that the standard Krylov method becomes more advantageous compared to RKSM as the outer iteration proceeds. The reason behind this is that the standard Krylov method is able to resolve the dominant eigenvectors $U_{j}$ of the solution $Y_{j}$ faster than RKSM in the case where $\angle\left(P_{j}, U_{j}\right)$ is already small. This point is demonstrated in Figure 5.5, which shows the decay of $\angle\left(\mathcal{K}, U_{j}\right)$, the angle between the subspace spanned by $U_{j}$, and the Krylov subspace. The decay curves in Figures 5.3 and 5.5 are clearly similar.

We conclude section 5.3 with the following remarks:

1. If the goal is to solve (3.1) accurately, RKSM is definitely the superior choice. Compared to the standard Krylov method, it has a faster asymptotic rate of convergence, which leads to significant savings in both storage and CPU time; moreover, the rank of the solution it computes is mesh-independent, which is important for problems arising from discretization of two- or threedimensional PDEs.

2. However, as pointed out in section 4, solving the Lyapunov equations accurately is not of primary interest in the current study, since we need only solve it accurately enough for the outer iteration, i.e., $\left\|R_{j}\right\|_{F}<\left\|\Re_{j}\right\|_{F}$ (see Algorithm 5, step 2.4 with $\delta=1$ ). In our experiments, $\left\|\mathfrak{R}_{1}\right\|_{F} \approx 3.81 \times 10^{2}$, $\left\|\mathfrak{R}_{2}\right\|_{F} \approx 1.52 \times 10^{-1},\left\|\mathfrak{R}_{3}\right\|_{F} \approx 2.35 \times 10^{-3}$, and $\left\|\mathfrak{R}_{4}\right\|_{F} \approx 7.17 \times 10^{-5}$ (see Table A.1 in Appendix A). Figure 5.3 shows that if the stopping criterion is this mild, the two methods require Krylov subspaces of almost the same dimension, and therefore RKSM will be the less effective choice between the two. 




(a) $j=1$

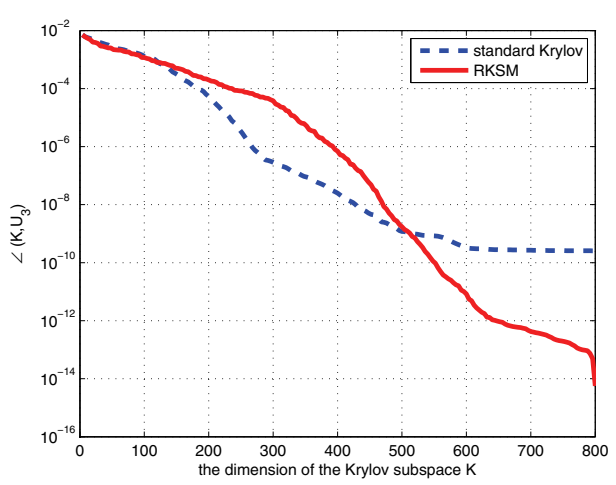

(c) $j=3$



(b) $j=2$

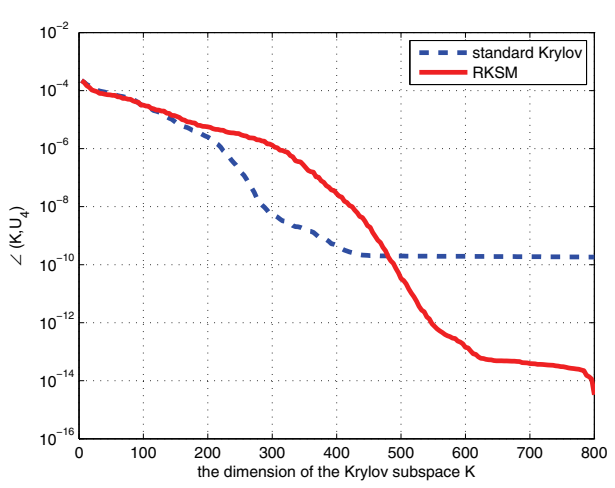

(d) $j=4$

Fig. 5.5. Decay of $\angle\left(\mathcal{K}, U_{j}\right)$ of the standard Krylov method and RKSM $(64 \times 64$ mesh $)$.

6. Conclusions. We have refined the Lyapunov inverse iteration proposed in [18] and examined the application of our algorithm to two examples arising from models of incompressible flow. The driven-cavity flow example is a particularly difficult problem. For both examples, the new algorithm is able to compute good estimates of the critical parameter value at which Hopf bifurcation takes place. Our algorithm belongs to the class of inner-outer iterative methods: the outer iteration is the inverse iteration for a special eigenvalue problem, and the inner iteration is to solve a Lyapunov equation. Based on existing theory of inner-outer iterative methods, the Lyapunov equations do not need to be solved to high accuracy; instead, a mild tolerance is sufficient. In this scenario, the standard Krylov method is as effective as RKSM for solving the large-scale Lyapunov systems that arise for our application.

\section{Appendix A. Numerical results for Example 1.}

A.1. The $64 \times 64$ mesh $(n=9539)$.

- Algorithm 5

- The IRA method: $7928 \leq R e^{*} \leq 7929$ and $\mu \approx \pm 2.69910 i$ 
TABLE A.1

Driven-cavity flow $\left(64 \times 64\right.$ mesh, $\left.R e_{0}=7700\right)$ for $\delta=1,10^{-1}, 10^{-2}$ in (4.4).

\begin{tabular}{|c|c|c|c|c|c|c|c|}
\hline$j$ & $R e_{j}$ & $\mu_{j}$ & $\left\|r_{j}\right\|_{2}$ & $\left\|\Re_{j}\right\|_{F}$ & $\left\|R_{j}\right\|_{F}$ & $m_{j}$ & $k_{j}$ \\
\hline & & \multicolumn{6}{|c|}{$\delta=1$} \\
\hline 1 & -33 & $-1.65191 \mathrm{e}-13$ & $3.11813 \mathrm{e}-01$ & $3.81288 \mathrm{e}+2$ & $3.49287 \mathrm{e}+2$ & 126 & 40 \\
\hline 2 & 8071 & $2.75632 \mathrm{i}$ & $2.28205 \mathrm{e}-04$ & $1.51762 \mathrm{e}-1$ & $1.51102 \mathrm{e}-1$ & 468 & 170 \\
\hline 3 & 7956 & $2.69951 \mathrm{i}$ & $5.94238 \mathrm{e}-06$ & $2.35053 \mathrm{e}-3$ & $2.29411 \mathrm{e}-3$ & 464 & 170 \\
\hline 4 & 7941 & $\overline{2.69869 i}$ & $1.25554 \mathrm{e}-07$ & $7.17013 \mathrm{e}-5$ & $6.92490 \mathrm{e}-5$ & 440 & 160 \\
\hline 5 & 7941 & $2.69871 \mathrm{i}$ & $5.22034 \mathrm{e}-09$ & $2.22796 \mathrm{e}-6$ & $2.11931 \mathrm{e}-6$ & 456 & 160 \\
\hline \multirow[t]{3}{*}{6} & 7941 & $2.69871 \mathrm{i}$ & $1.58703 \mathrm{e}-10$ & $7.03833 \mathrm{e}-8$ & - & - & - \\
\hline & & & & & Total: & 1954 & \\
\hline & & \multicolumn{6}{|c|}{$\delta=10^{-1}$} \\
\hline 1 & -33 & $-1.65191 \mathrm{e}-13$ & $3.11813 \mathrm{e}-01$ & $3.81288 \mathrm{e}+2$ & $3.67018 \mathrm{e}+1$ & 184 & 60 \\
\hline 2 & 8099 & $3.22383 \mathrm{i}$ & $3.63286 \mathrm{e}-04$ & $7.42002 \mathrm{e}-2$ & $7.21658 \mathrm{e}-3$ & 828 & 260 \\
\hline 3 & 8272 & $2.69587 \mathrm{i}$ & $3.06514 \mathrm{e}-05$ & $2.30574 \mathrm{e}-2$ & $2.29802 \mathrm{e}-3$ & 584 & 210 \\
\hline 4 & 7940 & $2.69870 \mathrm{i}$ & $9.47243 \mathrm{e}-07$ & $3.99983 \mathrm{e}-4$ & $3.99561 \mathrm{e}-5$ & 628 & 240 \\
\hline 5 & 7941 & $2.69871 \mathrm{i}$ & $3.10614 \mathrm{e}-08$ & $1.77510 \mathrm{e}-5$ & $1.74859 \mathrm{e}-6$ & 584 & 210 \\
\hline \multirow[t]{3}{*}{6} & 7941 & $2.69871 \mathrm{i}$ & $9.92136 \mathrm{e}-10$ & $6.43387 \mathrm{e}-7$ & $\overline{-}$ & - & $\overline{-}$ \\
\hline & & & & & Total: & 2808 & \\
\hline & & \multicolumn{6}{|c|}{$\delta=10^{-2}$} \\
\hline 1 & -33 & $-1.65191 \mathrm{e}-13$ & $3.11813 \mathrm{e}-01$ & $3.81288 \mathrm{e}+2$ & $3.75975 \mathrm{e}+0$ & 458 & 130 \\
\hline 2 & 8266 & $2.69436 \mathrm{i}$ & $3.23204 \mathrm{e}-05$ & $3.91517 \mathrm{e}-2$ & $3.74950 \mathrm{e}-4$ & 736 & 240 \\
\hline 3 & 7934 & $2.69853 \mathrm{i}$ & $5.63398 \mathrm{e}-07$ & $4.91929 \mathrm{e}-4$ & $4.79841 \mathrm{e}-6$ & 812 & 270 \\
\hline 4 & 7941 & $2.69872 \mathrm{i}$ & $3.36650 \mathrm{e}-08$ & $2.62097 \mathrm{e}-5$ & $2.53300 \mathrm{e}-7$ & 804 & 270 \\
\hline 5 & 7941 & $2.69871 \mathrm{i}$ & $2.06884 \mathrm{e}-09$ & $7.55187 \mathrm{e}-7$ & $7.36075 \mathrm{e}-9$ & 872 & 290 \\
\hline \multirow[t]{2}{*}{6} & 7941 & $2.69871 \mathrm{i}$ & $4.18021 \mathrm{e}-11$ & $2.58556 \mathrm{e}-8$ & - & - & - \\
\hline & & & & & Total: & 3682 & \\
\hline
\end{tabular}

TABLE A.2

Driven-cavity flow $\left(128 \times 128\right.$ mesh, $\left.R e_{0}=7900\right)$ for $\delta=1,10^{-1}, 10^{-2}$ in (4.4).

\begin{tabular}{cccccccc}
\hline$j$ & $R e_{j}$ & $\mu_{j}$ & $\left\|r_{j}\right\|_{2}$ & $\left\|\mathfrak{R}_{j}\right\|_{F}$ & $\left\|R_{j}\right\|_{F}$ & $m_{j}$ & $k_{j}$ \\
\hline & & & & & & \\
\hline 1 & -84 & $4.52639 \mathrm{e}-14$ & $1.98235 \mathrm{e}-01$ & $1.80556 \mathrm{e}+2$ & $1.46777 \mathrm{e}+2$ & 192 & 60 \\
\hline 2 & 7980 & $2.77602 \mathrm{i}$ & $1.49742 \mathrm{e}-05$ & $1.11252 \mathrm{e}-2$ & $1.08771 \mathrm{e}-2$ & 452 & 160 \\
\hline 3 & 8178 & $2.76959 \mathrm{i}$ & $3.59421 \mathrm{e}-07$ & $6.67463 \mathrm{e}-4$ & $6.60235 \mathrm{e}-4$ & 456 & 170 \\
\hline 4 & 8170 & $2.76985 \mathrm{i}$ & $1.52871 \mathrm{e}-08$ & $1.98948 \mathrm{e}-5$ & $1.77490 \mathrm{e}-5$ & 456 & 170 \\
\hline 5 & 8170 & $2.76986 \mathrm{i}$ & $5.65144 \mathrm{e}-10$ & $1.09343 \mathrm{e}-6$ & - & - & - \\
\hline & & & & Total: & 1556 & \\
\hline & & & $\delta=10^{-1}$ & & & \\
\hline 1 & -84 & $4.52639 \mathrm{e}-14$ & $1.98235 \mathrm{e}-01$ & $1.80556 \mathrm{e}+2$ & $1.80487 \mathrm{e}+1$ & 394 & 120 \\
\hline 2 & 8712 & $2.78474 \mathrm{i}$ & $1.28825 \mathrm{e}-05$ & $5.36405 \mathrm{e}-2$ & $4.89405 \mathrm{e}-3$ & 516 & 190 \\
\hline 3 & 8195 & $2.76859 \mathrm{i}$ & $3.60491 \mathrm{e}-07$ & $1.11571 \mathrm{e}-3$ & $1.06377 \mathrm{e}-4$ & 540 & 200 \\
\hline 4 & 8170 & $2.76988 \mathrm{i}$ & $2.75212 \mathrm{e}-08$ & $4.87574 \mathrm{e}-5$ & $4.57568 \mathrm{e}-6$ & 576 & 220 \\
\hline 5 & 8170 & $2.76986 \mathrm{i}$ & $7.86850 \mathrm{e}-10$ & $1.66872 \mathrm{e}-6$ & - & - & - \\
\hline & & & & & Total: & 2026 & \\
\hline & & & $\delta=10^{-2}$ & & & \\
\hline 1 & -84 & $4.52639 \mathrm{e}-14$ & $1.98235 \mathrm{e}-01$ & $1.80556 \mathrm{e}+2$ & $1.77080 \mathrm{e}+0$ & 552 & 150 \\
\hline 2 & 8279 & $2.78261 \mathrm{i}$ & $6.89377 \mathrm{e}-06$ & $2.57644 \mathrm{e}-2$ & $2.56827 \mathrm{e}-4$ & 732 & 270 \\
\hline 3 & 8183 & $2.76926 \mathrm{i}$ & $2.69987 \mathrm{e}-07$ & $3.79368 \mathrm{e}-4$ & $3.75942 \mathrm{e}-6$ & 784 & 280 \\
\hline 4 & 8171 & $2.76983 \mathrm{i}$ & $1.97231 \mathrm{e}-08$ & $2.36808 \mathrm{e}-5$ & $2.30965 \mathrm{e}-7$ & 764 & 270 \\
\hline 5 & 8170 & $2.76986 \mathrm{i}$ & $9.31122 \mathrm{e}-10$ & $1.88047 \mathrm{e}-6$ & - & - & - \\
\hline & & & & & & \\
\hline
\end{tabular}

Copyright (c) by SIAM. Unauthorized reproduction of this article is prohibited. 
A.2. The $128 \times 128$ mesh $(n=37507)$.

- Algorithm 5

- The IRA method: $8167 \leq R e^{*} \leq 8168$ and $\mu \approx \pm 2.76931 i$

\section{Appendix B. Numerical results for Example 2.}

B.1. The $32 \times 128 \operatorname{mesh}(n=9512)$.

- Algorithm 5

- The IRA method: $366 \leq R e^{*} \leq 367$ and $\mu \approx \pm 2.25320 i$

TABLE B. 1

Flow over an obstacle $\left(32 \times 128\right.$ mesh, $\left.R e_{0}=320\right)$ for $\delta=1,10^{-1}, 10^{-2}$ in (4.4).

\begin{tabular}{|c|c|c|c|c|c|c|c|}
\hline$j$ & $R e_{j}$ & $\mu_{j}$ & $\left\|r_{j}\right\|_{2}$ & $\left\|\Re_{j}\right\|_{F}$ & $\left\|R_{j}\right\|_{F}$ & $m_{j}$ & $k_{j}$ \\
\hline & & \multicolumn{6}{|c|}{$\delta=1$} \\
\hline 1 & -51 & $-2.58005 \mathrm{e}-14$ & $3.49466 \mathrm{e}-01$ & $1.34437 \mathrm{e}+1$ & $1.28722 \mathrm{e}+1$ & 64 & 10 \\
\hline 2 & 312 & $2.24624 \mathrm{i}$ & $1.03971 \mathrm{e}-03$ & $8.86864 \mathrm{e}-2$ & $8.81434 \mathrm{e}-2$ & 68 & 30 \\
\hline 3 & 372 & $2.25768 \mathrm{i}$ & $1.58031 \mathrm{e}-04$ & $5.38592 \mathrm{e}-3$ & $4.49832 \mathrm{e}-3$ & 68 & 30 \\
\hline 4 & 368 & $2.25509 \mathrm{i}$ & $4.52672 \mathrm{e}-05$ & $5.17687 \mathrm{e}-4$ & $4.22587 \mathrm{e}-4$ & 68 & 30 \\
\hline 5 & 368 & $2.25445 \mathrm{i}$ & $4.69228 \mathrm{e}-06$ & $6.75943 \mathrm{e}-5$ & $6.49335 \mathrm{e}-5$ & 68 & 30 \\
\hline 6 & 368 & $2.25466 \mathrm{i}$ & $6.44924 \mathrm{e}-07$ & $8.61572 \mathrm{e}-6$ & $3.78203 \mathrm{e}-6$ & 72 & 30 \\
\hline 7 & 368 & $2.25466 \mathrm{i}$ & $8.89108 \mathrm{e}-08$ & $1.56019 \mathrm{e}-6$ & $9.25999 \mathrm{e}-7$ & 72 & 30 \\
\hline 8 & 368 & $2.25466 \mathrm{i}$ & $3.34159 \mathrm{e}-08$ & $4.92662 \mathrm{e}-7$ & $3.60198 \mathrm{e}-7$ & 68 & 30 \\
\hline 9 & 368 & $2.25466 \mathrm{i}$ & $4.12733 \mathrm{e}-09$ & $7.20820 \mathrm{e}-8$ & $6.38617 \mathrm{e}-8$ & 68 & 30 \\
\hline 10 & 368 & $2.25466 \mathrm{i}$ & $1.52598 \mathrm{e}-09$ & $2.22553 \mathrm{e}-8$ & $1.25189 \mathrm{e}-8$ & 68 & 30 \\
\hline \multirow[t]{3}{*}{11} & 368 & $2.25466 \mathrm{i}$ & $2.521173-10$ & $3.57852 \mathrm{e}-9$ & - & - & - \\
\hline & & & & & Total: & 684 & \\
\hline & & \multicolumn{6}{|c|}{$\delta=10^{-1}$} \\
\hline 1 & -51 & $-2.58005 \mathrm{e}-14$ & $3.49466 \mathrm{e}-01$ & $1.34437 \mathrm{e}+1$ & $1.097413+0$ & 80 & 30 \\
\hline 2 & 340 & $2.25959 \mathrm{i}$ & $1.34391 \mathrm{e}-03$ & $1.24111 \mathrm{e}-1$ & $9.25966 \mathrm{e}-3$ & 80 & 30 \\
\hline 3 & 371 & $2.25850 \mathrm{i}$ & $2.84080 \mathrm{e}-04$ & $4.17974 \mathrm{e}-3$ & $3.65068 \mathrm{e}-4$ & 88 & 40 \\
\hline 4 & 368 & $2.25419 \mathrm{i}$ & $2.91437 \mathrm{e}-05$ & $3.48382 \mathrm{e}-4$ & $3.14857 \mathrm{e}-5$ & 84 & 40 \\
\hline 5 & 368 & $2.25459 \mathrm{i}$ & $1.66116 \mathrm{e}-06$ & $3.70041 \mathrm{e}-5$ & $3.63305 \mathrm{e}-6$ & 84 & 50 \\
\hline 6 & 368 & $2.25470 \mathrm{i}$ & $2.05981 \mathrm{e}-07$ & $8.67526 \mathrm{e}-6$ & $6.08886 \mathrm{e}-7$ & 84 & 40 \\
\hline 7 & 368 & $2.25466 \mathrm{i}$ & $1.20058 \mathrm{e}-07$ & $1.98096 \mathrm{e}-6$ & $1.73313 \mathrm{e}-7$ & 84 & 40 \\
\hline 8 & 368 & $2.25466 \mathrm{i}$ & $1.92445 \mathrm{e}-08$ & $4.79664 \mathrm{e}-7$ & $4.44614 \mathrm{e}-8$ & 80 & 40 \\
\hline 9 & 368 & $2.25466 \mathrm{i}$ & $4.90524 \mathrm{e}-09$ & $7.95222 \mathrm{e}-8$ & $7.07355 \mathrm{e}-9$ & 84 & 40 \\
\hline \multirow[t]{3}{*}{10} & 368 & $2.25466 \mathrm{i}$ & $7.62478 \mathrm{e}-10$ & $1.64741 \mathrm{e}-8$ & - & - & - \\
\hline & & & & & Total: & 748 & \\
\hline & & \multicolumn{6}{|c|}{$\delta=10^{-2}$} \\
\hline 1 & -51 & $-2.58005 \mathrm{e}-14$ & $3.49466 \mathrm{e}-01$ & $1.34437 \mathrm{e}+1$ & $1.33974 \mathrm{e}-1$ & 256 & 50 \\
\hline 2 & 355 & $2.23301 \mathrm{i}$ & $5.20369 \mathrm{e}-04$ & $1.27854 \mathrm{e}-2$ & $1.27569 \mathrm{e}-4$ & 212 & 80 \\
\hline 3 & 368 & $2.25539 \mathrm{i}$ & $1.16498 \mathrm{e}-04$ & $1.68074 \mathrm{e}-3$ & $1.55230 \mathrm{e}-5$ & 184 & 60 \\
\hline 4 & 368 & $2.25479 \mathrm{i}$ & $1.25632 \mathrm{e}-05$ & $1.71052 \mathrm{e}-4$ & $1.63911 \mathrm{e}-6$ & 180 & 60 \\
\hline 5 & 368 & $2.25465 \mathrm{i}$ & $5.29902 \mathrm{e}-07$ & $1.04054 \mathrm{e}-5$ & $1.02432 \mathrm{e}-7$ & 192 & 70 \\
\hline 6 & 368 & $2.25466 \mathrm{i}$ & $5.71101 \mathrm{e}-08$ & $1.09042 \mathrm{e}-6$ & $1.06047 \mathrm{e}-8$ & 172 & 60 \\
\hline 7 & 368 & $2.25466 \mathrm{i}$ & $6.17975 \mathrm{e}-09$ & $1.37103 \mathrm{e}-7$ & $1.35858 \mathrm{e}-9$ & 180 & 60 \\
\hline 8 & 368 & $2.25466 \mathrm{i}$ & $8.80752 \mathrm{e}-10$ & $2.09028 \mathrm{e}-8$ & - & - & - \\
\hline & & & & & Total: & 1376 & \\
\hline
\end{tabular}

B.2. The $64 \times 256$ mesh $(n=37168)$.

- Algorithm 5

- The IRA method: $371 \leq R e^{*} \leq 372$ and $\mu \approx \pm 2.26399 i$

Copyright $($ C by SIAM. Unauthorized reproduction of this article is prohibited. 
TABLE B.2

Flow over an obstacle $\left(64 \times 256\right.$ mesh, $\left.\operatorname{Re}_{0}=320\right)$ for $\delta=1,10^{-1}, 10^{-2}$ in (4.4).

\begin{tabular}{|c|c|c|c|c|c|c|c|}
\hline$j$ & $R e_{j}$ & $\mu_{j}$ & $\left\|r_{j}\right\|_{2}$ & $\left\|\Re_{j}\right\|_{F}$ & $\left\|R_{j}\right\|_{F}$ & $m_{j}$ & $k_{j}$ \\
\hline & & \multicolumn{6}{|c|}{$\delta=1$} \\
\hline 1 & -126 & $-4.56508 \mathrm{e}-14$ & $2.34469 \mathrm{e}-01$ & $6.82009 \mathrm{e}+0$ & $6.54563 \mathrm{e}+0$ & 68 & 10 \\
\hline 2 & 309 & $2.25503 \mathrm{i}$ & $1.22214 \mathrm{e}-03$ & $2.67894 \mathrm{e}-1$ & $1.47060 \mathrm{e}-1$ & 60 & 10 \\
\hline 3 & 388 & $2.28406 \mathrm{i}$ & $1.79532 \mathrm{e}-04$ & $1.44874 \mathrm{e}-2$ & $1.38221 \mathrm{e}-2$ & 68 & 30 \\
\hline 4 & 371 & $2.26307 \mathrm{i}$ & $3.24286 \mathrm{e}-05$ & $1.50971 \mathrm{e}-3$ & $7.80000 \mathrm{e}-4$ & 68 & 30 \\
\hline 5 & 372 & $2.26454 \mathrm{i}$ & $2.80872 \mathrm{e}-06$ & $1.26709 \mathrm{e}-4$ & $8.29876 \mathrm{e}-5$ & 68 & 20 \\
\hline 6 & 372 & $2.26439 \mathrm{i}$ & $1.35033 \mathrm{e}-06$ & $5.10769 \mathrm{e}-5$ & $3.90744 \mathrm{e}-5$ & 64 & 20 \\
\hline 7 & 372 & $2.26440 \mathrm{i}$ & $1.15382 \mathrm{e}-07$ & $5.09132 \mathrm{e}-6$ & $4.22834 \mathrm{e}-6$ & 68 & 30 \\
\hline 8 & 372 & $2.26441 \mathrm{i}$ & $5.60313 \mathrm{e}-08$ & $2.03776 \mathrm{e}-6$ & $1.31217 \mathrm{e}-6$ & 64 & 20 \\
\hline 9 & 372 & $2.26441 \mathrm{i}$ & $4.75726 \mathrm{e}-09$ & $2.25479 \mathrm{e}-7$ & $1.17966 \mathrm{e}-7$ & 68 & 20 \\
\hline 10 & 372 & $2.26441 \mathrm{i}$ & $1.91912 \mathrm{e}-09$ & $6.90577 \mathrm{e}-8$ & $3.15263 \mathrm{e}-8$ & 64 & 20 \\
\hline \multirow[t]{3}{*}{11} & 372 & $2.26441 \mathrm{i}$ & $2.16132 \mathrm{e}-10$ & $8.88705 \mathrm{e}-9$ & - & - & - \\
\hline & & & & & Total: & 660 & \\
\hline & & \multicolumn{6}{|c|}{$\delta=10^{-1}$} \\
\hline 1 & -126 & $-4.56508 \mathrm{e}-14$ & $2.34469 \mathrm{e}-01$ & $6.82009 \mathrm{e}+0$ & $5.94744 \mathrm{e}-1$ & 200 & 30 \\
\hline 2 & 371 & $2.24869 \mathrm{i}$ & $5.87893 \mathrm{e}-04$ & $4.21899 \mathrm{e}-2$ & $4.09704 \mathrm{e}-3$ & 92 & 40 \\
\hline 3 & 369 & $2.26101 \mathrm{i}$ & $8.09361 \mathrm{e}-05$ & $3.07705 \mathrm{e}-3$ & $2.78703 \mathrm{e}-4$ & 80 & 40 \\
\hline 4 & 372 & $2.26488 \mathrm{i}$ & $8.09872 \mathrm{e}-06$ & $3.53202 \mathrm{e}-4$ & $2.66546 \mathrm{e}-5$ & 80 & 40 \\
\hline 5 & 372 & $2.26446 \mathrm{i}$ & $5.68983 \mathrm{e}-07$ & $3.12131 \mathrm{e}-5$ & $1.81808 \mathrm{e}-6$ & 84 & 40 \\
\hline 6 & 372 & $2.26440 \mathrm{i}$ & $5.69946 \mathrm{e}-08$ & $3.94377 \mathrm{e}-6$ & $3.11348 \mathrm{e}-7$ & 84 & 40 \\
\hline 7 & 372 & $2.26441 \mathrm{i}$ & $2.64065 \mathrm{e}-08$ & $1.19487 \mathrm{e}-6$ & $8.67071 \mathrm{e}-8$ & 80 & 40 \\
\hline 8 & 372 & $2.26441 \mathrm{i}$ & $4.71386 \mathrm{e}-09$ & $1.98825 \mathrm{e}-7$ & $1.20088 \mathrm{e}-8$ & 80 & 40 \\
\hline \multirow[t]{3}{*}{9} & 372 & $2.26441 \mathrm{i}$ & $6.18183 \mathrm{e}-10$ & $2.82239 \mathrm{e}-9$ & - & - & - \\
\hline & & & & & Total: & 780 & \\
\hline & & \multicolumn{6}{|c|}{$\delta=10^{-2}$} \\
\hline 1 & -126 & $-4.56508 \mathrm{e}-14$ & $2.34469 \mathrm{e}-01$ & $6.82009 \mathrm{e}+0$ & $5.65414 \mathrm{e}-2$ & 254 & 50 \\
\hline 2 & 355 & $2.24026 \mathrm{i}$ & $1.94550 \mathrm{e}-04$ & $1.49911 \mathrm{e}-2$ & $1.31541 \mathrm{e}-4$ & 184 & 60 \\
\hline 3 & 370 & $2.26957 \mathrm{i}$ & $7.74197 \mathrm{e}-05$ & $3.01197 \mathrm{e}-3$ & $2.89703 \mathrm{e}-5$ & 152 & 60 \\
\hline 4 & 372 & $2.26422 \mathrm{i}$ & $1.07494 \mathrm{e}-05$ & $4.01064 \mathrm{e}-4$ & $3.94011 \mathrm{e}-6$ & 156 & 60 \\
\hline 5 & 372 & $2.26440 \mathrm{i}$ & $1.55387 \mathrm{e}-06$ & $5.52818 \mathrm{e}-5$ & $5.09448 \mathrm{e}-7$ & 156 & 50 \\
\hline 6 & 372 & $2.26442 \mathrm{i}$ & $4.72673 \mathrm{e}-08$ & $2.39237 \mathrm{e}-6$ & $2.21539 \mathrm{e}-8$ & 168 & 60 \\
\hline 7 & 372 & $2.26441 \mathrm{i}$ & $6.31077 \mathrm{e}-09$ & $2.60586 \mathrm{e}-7$ & $2.24572 \mathrm{e}-9$ & 180 & 60 \\
\hline \multirow[t]{2}{*}{8} & 372 & $2.26441 \mathrm{i}$ & $9.64857 \mathrm{e}-10$ & $4.66424 \mathrm{e}-8$ & - & - & - \\
\hline & & & & & Total: & 1250 & \\
\hline
\end{tabular}

\section{REFERENCES}

[1] A. C. Antoulas, Approximation of Large-Scale Dynamical Systems, SIAM, Philadelphia, 2005.

[2] F. Auteri, N. Parolini, and L. Quartapelle, Numerical investigation on the stability of singular driven cavity flow, J. Comput. Phys., 183 (2002), pp. 1-25.

[3] R. H. Bartels and G. W. Stewart, Algorithm 432: Solution of the matrix equation AX + $X B=C$, Comm. ACM, 15 (1972), pp. 820-826.

[4] C.-H. Bruneau and M. SaAd, The $2 D$ lid-driven cavity problem revisited, Comput. \& Fluids, 35 (2006), pp. 326-348.

[5] K. A. Cliffe, T. J. Garratt, and A. Spence, Eigenvalues of block matrices arising from problems in fluid mechanics, SIAM J. Matrix Anal. Appl., 15 (1994), pp. 1310-1318.

[6] V. Druskin, L. Knizhnerman, and V. Simoncini, Analysis of the rational Krylov subspace and ADI methods for solving the Lyapunov equation, SIAM J. Numer. Anal., 49 (2011), pp. $1875-1898$.

[7] V. Druskin, C. Lieberman, and M. Zaslavsky, On adaptive choice of shifts in rational Krylov subspace reduction of evolutionary problems, SIAM J. Sci. Comput., 32 (2010), pp. $2485-2496$.

[8] V. Druskin and V. Simoncini, Adaptive rational Krylov subspaces for large-scale dynamical systems, Systems Control Lett., 60 (2011), pp. 546-560.

[9] H. Elman, D. Silvester, and A. Wathen, Finite Elements and Fast Iterative Solvers, Oxford University Press, Oxford, UK, 2005.

Copyright (C) by SIAM. Unauthorized reproduction of this article is prohibited. 
[10] A. Fortin, M. Jardak, And J. Gervais, Localization of Hopf bifurcation in fluid flow problems, Internat. J. Numer. Methods Fluids, 24 (1997), pp. 1185-1210.

[11] J. J. Gervais, D. Lemelin, and R. Pierre, Some experiments with stability analysis of discrete incompressible flows in the lid-driven cavity, Internat. J. Numer. Methods Fluids, 24 (1997), pp. 477-492.

[12] W. J. F. Govaerts, Numerical Methods for Bifurcations of Dynamical Equilibria, SIAM, Philadelphia, 2000.

[13] J. Guckenheimer And M. Myers, Computing Hopf bifurcations II: Three examples from neurophysiology, SIAM J. Sci. Comput., 17 (1996), pp. 1275-1301.

[14] J. GuCKenheimer, M. Myers, and B. Sturmfels, Computing Hopf bifurcations I, SIAM J. Numer. Anal., 34 (1997), pp. 1-21.

[15] S. J. Hammarling, Numerical solution of the stable, non-negative definite Lyapunov equation, IMA J. Numer. Anal., 2 (1982), pp. 303-323.

[16] R. A. Horn and C. R. Johnson, Topics in Matrix Analysis, Cambridge University Press, Cambridge, UK, 1991.

[17] I. M. Jaimoukha AND E. M. KASEnAlLy, Krylov subspace methods for solving large Lyapunov equations, SIAM J. Numer. Anal., 31 (1994), pp. 227-251.

[18] K. Meerbergen And A. Spence, Inverse iteration for purely imaginary eigenvalues with application to the detection of Hopf bifurcations in large-scale problems, SIAM J. Matrix Anal. Appl., 31 (2010), pp. 1982-1999.

[19] M. Robbé, M. Sadkane, And A. Spence, Inexact inverse subspace iteration with preconditioning applied to non-Hermitian eigenvalue problems, SIAM J. Matrix Anal. Appl., 31 (2009), pp. 92-113.

[20] A. RuHe, Rational Krylov sequence methods for eigenvalue computation, Linear Algebra Appl., 58 (1984), pp. 391-405.

[21] A. RuHe, The rational Krylov algorithm for nonsymmetric eigenvalue problems. III: Complex shifts for real matrices, BIT, 34 (1994), pp. 165-176.

[22] Y. SAAD, Numerical solution of large Lyapunov equations, in Signal Processing, Scattering, Operator Theory, and Numerical Methods, M. A. Kaashoek, J. H. van Schuppen, and A. C. Ran, eds., Birkhäuser, Boston, 1990, pp. 503-511.

[23] V. Simoncini, A new iterative method for solving large-scale Lyapunov matrix equations, SIAM J. Sci. Comput., 29 (2007), pp. 1268-1288.

[24] D. C. Sorensen, Implicit application of polynomial filters in a $k$-step Arnoldi method, SIAM J. Matrix Anal. Appl., 13 (1992), pp. 357-385.

[25] P. A. Wedin, On angles between subspaces of a finite dimensional inner product space, in Matrix Pencils, Lecture Notes in Math. 973, B. Kagstrom and A. Ruhe, eds., SpringerVerlag, Berlin, 1993, pp. 263-285.

Copyright (c) by SIAM. Unauthorized reproduction of this article is prohibited. 